\title{
LINEAR MODELS FOR COMPOSITE THIN-WALLED BEAMS BY $\Gamma-$ CONVERGENCE. PART II: CLOSED CROSS-SECTIONS
}

\author{
CESARE DAVINI, LORENZO FREDDI, AND ROBERTO PARONI
}

July 10, 2014

\begin{abstract}
We consider a beam whose cross-section is a tubular neighborhood of a simple closed curve $\gamma$. We assume that the wall thickness, i.e., the size of the neighborhood, scales with a parameter $\delta_{\varepsilon}$ while the length of $\gamma$ scales with $\varepsilon$. We characterize a thin-walled beam by assuming that $\delta_{\varepsilon}$ goes to zero faster than $\varepsilon$. Starting from the three dimensional linear theory of elasticity, by letting $\varepsilon$ go to zero, we derive a one-dimensional $\Gamma$-limit problem for the case in which the ratio between $\varepsilon^{2}$ and $\delta_{\varepsilon}$ is bounded. The limit model is obtained for a fully anisotropic and inhomogeneous material, thus making the theory applicable for composite thin-walled beams. Our approach recovers in a systematic way, and gives account of, many features of the beam models in the theory of Vlasov.
\end{abstract}

\section{INTRODUCTION}

The attention to thin-walled beams has been motivated by their peculiar torsional behavior. The pioneering works by Prandtl (1903) and Timoshenko (1905), see [10] and [11], on the flexural-torsional instability of beams opened the way to an extensive study of the subject that has occupied a large part of the first half of the last century. A fundamental contribution was given by Vlasov, whose work became known in the west at the end of the '50s after the English translation of his monograph [12].

The high structural performances and the low weight make thin-walled structures of great interest for applications, especially in advanced technological fields. An account of the developments of the theory and of the problems arising in classical structural contexts is found in [8]. Advanced applications are met, for instance, in aeronautics and turbomachinery. In those fields dynamical and stability aspects become crucial and have urged research to explore novel areas and problems. In particular, it is common to use new and fiber reinforced materials, or to resort to special structural arrangements, in order to influence or control the dynamical behavior [9]. All this has contributed to the growth of an abundant literature and to a multitude of models that are often hardly comparable [13].

Dipartimento di Ingegneria Civile ed Architettura, via Cotonificio 114, 33100 Udine, Italy, email: davini@uniud.it .

Dipartimento di Matematica e Informatica, via delle Scienze 206, 33100 Udine, Italy, email: freddi@dimi.uniud.it .

Dipartimento di Architettura e Pianificazione, Università degli Studi di Sassari, Palazzo del Pou Salit, Piazza Duomo, 07041 Alghero, Italy, email: paroni@uniss.it . 
The various models are based on ad hoc kinematical assumptions. A different approach is taken in $[5,6]$, where the authors deduce an asymptotic model for thinwalled beams by starting from the three dimensional theory of elasticity and using the general framework of $\Gamma$-convergence. These papers deal with homogeneous isotropic elastic beams with rectangular cross-section. Namely, it is assumed that the long side of the cross-section scales with a small parameter $\varepsilon$, while the other with $\varepsilon^{2}$. This different scaling leads to a compactness result in which the different components of the displacement scale differently. It is also noticeable that the kinematic restrictions on the limit displacement field, that are the starting point of many direct models, here follow by compactness.

There is now a wide literature on thin-walled beams with open cross-section via variational convergence. In particular, in [7] the authors consider the case of an inhomogeneous anisotropic rectangular cross-section, and in $[3,4]$ the analysis has been extended to the non linear context. Recently, Davoli [2] considered the case of homogeneous anisotropic beams with curved open cross-section within the framework of finite deformations. In that article, however, the analysis of $\Gamma$-convergence is carried on in terms of strains rather than displacements, as it is usually done. In [1] we have studied inhomogeneous anisotropic thin-walled beams with an open curved cross-section within the framework of linear elasticity. In particular, when applied to homogeneous and isotropic materials, our results validate Vlasov's theory.

Here we consider thin-walled beams with closed cross-section. As far as we know, this is the first paper that deals with this case within the framework of variational convergence.

The main difference between open and closed cross-sections is essentially due to the different torsional rigidity. The mechanical reason is that the flux of the shear stresses across the cords of a closed cross-section does not vanish, upraising by up to two orders of magnitude the contribution of the De Saint Venant's regime of stresses to the global torsional rigidity, see [8]. This fact implies that the effects of nonuniform torsion produced by loads applied to the ends of the beam die off much faster than for an open cross-section. Or, in other words, that the importance of "warping" is severely reduced.

In the present approach the difference between open and closed cross-sections emerges at the kinematic level and is related to the multiple connection of the cross-section. The requirement that the displacements fulfill periodicity conditions yields that additional information can be obtained from the compactness theorem. Accordingly, the kinematical analysis of [1] can be significantly enhanced. In particular, it is found that the twist used for an open cross-section, hereafter denoted by $\vartheta$, is identically equal to zero. This result essentially states that the sequence that generates the twist in the case of open cross-sections is too rough and needs to be refined in the case of closed cross-sections. Indeed, by introducing a further rescaling it is possible to define a new quantity, $\Theta^{\varepsilon}$, that describes the twist angle of the cross-section and that converges to a limit value $\Theta$. It is worth saying that, by definition, $\Theta^{\varepsilon}$ and then $\Theta$ depend also on the shape of the cross section, reflecting a well known technical feature of torsion.

The periodicity of the displacements plays a crucial role and leads to new integral constraints on the potentials appearing in the limit strains. As a consequence, the definition of the limit energy density is much more involved with respect to the 
case of an open cross-section. Within the framework of homogeneous and isotropic materials, one of these integral constraints leads to a formula upon which Vlasov's theory of sectorial areas for closed cross-sections is based on, see Remark 4.7 and equation (43).

The paper is organized as follows. In Sections 2 and 3 we introduce the problem and recall some useful formulas from [1]. After recalling the compactness results, in Section 4 we prove that the limit twist $\vartheta$, as we defined it for the open crosssections, vanishes and introduce a new measure of twist $\Theta^{\varepsilon}$. We also update the representation formulae for the limit strains and deduce the integral constraints of the related potential functions. In Section 5 we compute a lower bound of the energy by minimizing the strain energy functional in the class of strains characterized in the previous sections. By this procedure we obtain a reduced form of the energy that will define the energy density of the $\Gamma$-limit. In Section 6 we find the $\Gamma$-limit through the usual sequential characterization and in the following section we prove that the $\Gamma$-limit has a local form. Namely, we give an explicit representation of the associated strain energy density.

We stress once more that our approach applies to generally inhomogeneous anisotropic thin-walled beams. The availability of an explicit representation formula for the strain energy density of the asymptotic model can be useful to discuss, for instance, the optimal arrangement of anisotropies in specific problems, or to suggest strategies for structural tailoring in advanced technological devices, see [9]. Finally, by the sake of exemplification, in the last section we calculate the $\Gamma$-limit for a homogeneous isotropic elastic thin-walled beam.

\section{Notation}

We adopt the same notation used in [1], which we recall hereafter for convenience of the reader. In the proofs, for brevity, we neglect to specify which identities hold only "almost everywhere", while we are explicit in the statements of the theorems.

Throughout the article, and unless otherwise stated, we index vector and tensor components as follows: Greek indices $\alpha, \beta$ and $\gamma$ take values in the set $\{1,2\}$ and Latin indices $i, j, k, l$ in the set $\{1,2,3\}$. With $\left(e_{1}, e_{2}, e_{3}\right)$ we shall denote the canonical basis of $\mathbb{R}^{3} . L^{p}(A ; B)$ and $H^{s}(A ; B)$ are the standard Lebesgue and Sobolev spaces of functions defined on the domain $A$ and taking values in $B$. When $B=\mathbb{R}$, or when the target set $B$ is clear from the context, we will simply write $L^{p}(A)$ or $H^{s}(A)$; also in the norms we shall systematically drop the target set. Convergence in the norm, that is the so-called strong convergence, will be denoted by $\rightarrow$ while weak convergence is denoted with $\rightarrow$. With a little abuse of language, and because this is a common practice and does not give rise to any confusion, we use to call "sequences" even those families indicized by a continuous parameter $\varepsilon$ which, throughout the whole paper, will be assumed to belong to the interval $(0,1]$. Throughout the paper, the constant $C$ may change from expression to expression and, in some case, even in the same line. The scalar product between vectors or tensors is denoted by $\cdot \mathbb{R}_{\text {skw }}^{3 \times 3}$ denotes the vector space of skew-symmetric $3 \times 3$ real matrices. For $A=\left(a_{i j}\right) \in \mathbb{R}^{3 \times 3}$ we denote the Euclidean norm (with the summation convention) by $|A|=\sqrt{A \cdot A}=\sqrt{\operatorname{tr}\left(A A^{T}\right)}=\sqrt{a_{i j} a_{i j}}$. Whenever we write a matrix by means of its columns we separate the columns with vertical bars $(\cdot|\cdot| \cdot) \in \mathbb{R}^{3 \times 3} . \partial_{i}$ stands for the distributional derivative $\frac{\partial}{\partial x_{i}}$. For every $a, b \in \mathbb{R}^{3}$ we denote by $a \odot b:=\frac{1}{2}(a \otimes b+b \otimes a)$ the symmetrized diadic product, where 
$(a \otimes b)_{i j}=a_{i} b_{j}$. When a function of three variables is independent of one or two of them we consider it as a function of the remaining variables only. This means, for instance, that a function $u \in H^{1}\left((0, \ell) \times(0, L) ; \mathbb{R}^{m}\right)$ will be identified with a corresponding $u \in H^{1}\left((0, \ell) \times(-h / 2, h / 2) \times(0, L) ; \mathbb{R}^{m}\right)$ such that $\partial_{2} u=0$ and a function $v \in H^{1}\left((0, L) ; \mathbb{R}^{m}\right)$ will be identified with a corresponding $v \in$ $H^{1}\left((0, \ell) \times(-h / 2, h / 2) \times(0, L) ; \mathbb{R}^{m}\right)$ such that $\partial_{1} v=\partial_{2} v=0$. The notation $d a$ stands for the area element $d x_{1} d x_{2}$. As usual, $f$ denotes the integral mean value.

\section{Statement of The PRoblem}

We consider a sequence of thin-walled beams with closed cross-section $\hat{\omega}_{\varepsilon}$ that is an annular neighborhood of thickness $\delta_{\varepsilon} h$ of a curve $\varepsilon \gamma$. Precisely, let $\ell>0$ and $I=[0, \ell] \subset \mathbb{R}$ be a closed interval and $\gamma: I \rightarrow \mathbb{R}^{2} \times\{0\}$ be a regular simple curve of length $\ell$ in the plane $x_{3}=0$. We shall assume that $\gamma$ is of class $W^{3, \infty}$ on the torus $[0, \ell]$. Then, the cross-section $\hat{\omega}_{\varepsilon}$ consists of the set of points

$$
\hat{x}=\varepsilon \gamma\left(x_{1}\right)+\delta_{\varepsilon} x_{2} n\left(x_{1}\right), \quad x_{1} \in I, x_{2} \in\left(-\frac{h}{2}, \frac{h}{2}\right),
$$

with $h>0$. Here $x_{1}$ is the arc length of $\gamma$ and $x_{2}$ measures the distance of point $\hat{x}$ from the mean curve $\varepsilon \gamma$ along the normal $n\left(x_{1}\right)$. We denote by $t:=\partial_{1} \gamma$ the unit tangent to $\gamma$ and by $n=e_{3} \wedge t$ the unit normal. In particular, $\kappa:=\partial_{1} t \cdot n$ is the curvature of $\gamma$, and we have that $\partial_{1} t=\kappa n$ and $\partial_{1} n=-\kappa t$. Clearly, $\kappa$ cannot be identically equal to zero because the curve is closed.

As in [1], we assume that the cross-section is thin in the sense established by

$$
\lim _{\varepsilon \rightarrow 0} \frac{\delta_{\varepsilon}}{\varepsilon}=0 .
$$

Let $\widehat{\Omega}_{\varepsilon}:=\hat{\omega}_{\varepsilon} \times(0, L)$ denote the domain occupied by the beam in the reference configuration. We indicate by

$$
E \hat{u}(\hat{x}):=\operatorname{sym}(\nabla \hat{u}(\hat{x})):=\frac{\nabla \hat{u}(\hat{x})+\nabla \hat{u}(\hat{x})^{T}}{2},
$$

the strain corresponding to the displacement $\hat{u}: \widehat{\Omega}_{\varepsilon} \rightarrow \mathbb{R}^{3}$.

We consider thin-walled beams made of an inhomogeneous linear hyper-elastic material characterized by an elasticity tensor $\mathbb{C}^{\varepsilon}$ with components $\mathbb{C}_{i j k l}^{\varepsilon} \in L^{\infty}\left(\widehat{\Omega}_{\varepsilon}\right)$ and satisfying the major and minor symmetries, i.e., $\mathbb{C}_{i j k l}^{\varepsilon}=\mathbb{C}_{i j l k}^{\varepsilon}=\mathbb{C}_{k l i j}^{\varepsilon}$. The fourth order tensor $\mathbb{C}^{\varepsilon}$ is assumed to be uniformly positive definite, that is: there exists $c>0$ such that

$$
\mathbb{C}^{\varepsilon}(\hat{x}) E \cdot E \geq c|E|^{2}
$$

for almost every $\hat{x}$, for all symmetric matrices $E$, and for every $\varepsilon>0$.

We take the beam to be clamped at $x_{3}=0$, and denote by

$$
H_{d n}^{1}\left(\widehat{\Omega}_{\varepsilon} ; \mathbb{R}^{3}\right):=\left\{\hat{u} \in H^{1}\left(\widehat{\Omega}_{\varepsilon} ; \mathbb{R}^{3}\right): \hat{u}=0 \text { on } \hat{\omega}_{\varepsilon} \times\{0\}\right\}
$$

the function space of the displacement fields. Then, the energy functional of the beam $\hat{\mathscr{F}}_{\varepsilon}: H_{d n}^{1}\left(\widehat{\Omega}_{\varepsilon} ; \mathbb{R}^{3}\right) \rightarrow \mathbb{R}$ is given by

$$
\hat{\mathscr{F}}_{\varepsilon}(\hat{u}):=\frac{1}{2} \int_{\widehat{\Omega}_{\varepsilon}} \mathbb{C}^{\varepsilon} E \hat{u} \cdot E \hat{u} d \hat{x}-\hat{\mathscr{L}}_{\varepsilon}(\hat{u})
$$

where $\hat{\mathscr{L}}_{\varepsilon}(\hat{u})$ denotes the work done by the loads on the displacements $\hat{u}$. 
We aim to find a variational limit of the functionals $\hat{\mathscr{F}}_{\varepsilon}$, as $\varepsilon \rightarrow 0$, in order to get an asymptotic model for thin-walled beams with closed cross-section. The analysis will be focused on the asymptotic behavior of the elastic energy only, and the reader is referred back to the considerations made in [1], Remark 7.3, for what concerns the work done by the loads.

\section{Problem on a fiXed domain}

According to (1), let

$$
\hat{x}=\chi_{\varepsilon}(x):=\varepsilon \gamma\left(x_{1}\right)+\delta_{\varepsilon} x_{2} n\left(x_{1}\right)+x_{3} e_{3} .
$$

be the function that maps the fixed domain $[0, \ell] \times\left(-\frac{h}{2}, \frac{h}{2}\right) \times(0, L)$ to the domain $\widehat{\Omega}_{\varepsilon}$ occupied by the beam in the reference configuration.

By means of $\chi_{\varepsilon}$ it is possible to write the mechanical problem on a fixed domain by defining

$$
u:=\hat{u} \circ \chi_{\varepsilon} .
$$

The chain rule yields then

$$
\nabla u=\nabla \hat{u} \circ \chi_{\varepsilon} \nabla \chi_{\varepsilon}
$$

where $\nabla u=\left(\partial_{1} u\left|\partial_{2} u\right| \partial_{3} u\right)$. From (7) it follows that

$$
\nabla \chi_{\varepsilon}=\left(\varepsilon\left(1-\frac{\delta_{\varepsilon}}{\varepsilon} x_{2} \kappa\right) t\left(x_{1}\right)\left|\delta_{\varepsilon} n\left(x_{1}\right)\right| e_{3}\right),
$$

hence

$$
\nabla \hat{u}=\nabla u\left(\nabla \chi_{\varepsilon}\right)^{-1}=\nabla u\left(\frac{1}{\varepsilon\left(1-\frac{\delta_{\varepsilon}}{\varepsilon} x_{2} \kappa\right)} t\left(x_{1}\right)\left|\frac{1}{\delta_{\varepsilon}} n\left(x_{1}\right)\right| e_{3}\right)^{T} .
$$

It is convenient for the sequel to express the deformation gradient and the strain tensor in a local frame. To this aim, at the points of $\widehat{\Omega}_{\varepsilon}$, or equivalently at the corresponding points of $[0, \ell] \times\left(-\frac{h}{2}, \frac{h}{2}\right) \times(0, L)$, we introduce the basis

$$
g_{1}^{\varepsilon}=\left(1-\frac{\delta_{\varepsilon}}{\varepsilon} x_{2} \kappa\right) t, \quad g_{2}^{\varepsilon}=n, \quad g_{3}^{\varepsilon}=e_{3},
$$

and its dual one

$$
g_{\varepsilon}^{1}=\frac{t}{1-\frac{\delta_{\varepsilon}}{\varepsilon} x_{2} \kappa}, \quad g_{\varepsilon}^{2}=n, \quad g_{\varepsilon}^{3}=e_{3} .
$$

We write $H^{\varepsilon} u$ and $E^{\varepsilon} u$ respectively for the deformation gradient and the strain tensor when they are regarded as fields over the fixed domain, that is

$$
H^{\varepsilon} u:=\left(\frac{1}{\varepsilon} \partial_{1} u\left|\frac{1}{\delta_{\varepsilon}} \partial_{2} u\right| \partial_{3} u\right)\left(g_{\varepsilon}^{1}\left|g_{\varepsilon}^{2}\right| g_{\varepsilon}^{3}\right)^{T},
$$

whereas $E^{\varepsilon} u$ is given by

$$
E^{\varepsilon} u:=\operatorname{sym} H^{\varepsilon} u .
$$

We note that (12) implies that

$$
\left(\frac{1}{\varepsilon} \partial_{1} u\left|\frac{1}{\delta_{\varepsilon}} \partial_{2} u\right| \partial_{3} u\right)=H^{\varepsilon} u\left(g_{1}^{\varepsilon}\left|g_{2}^{\varepsilon}\right| g_{3}^{\varepsilon}\right),
$$

that is,

$$
\frac{1}{\varepsilon} \partial_{1} u=H^{\varepsilon} u g_{1}^{\varepsilon}, \quad \frac{1}{\delta_{\varepsilon}} \partial_{2} u=H^{\varepsilon} u g_{2}^{\varepsilon}, \quad \partial_{3} u=H^{\varepsilon} u g_{3}^{\varepsilon} .
$$


We also notice that the components of $H^{\varepsilon} u$ in the local basis

$$
\left(H^{\varepsilon} u\right)_{i j}:=g_{i}^{\varepsilon} \cdot H^{\varepsilon} u g_{j}^{\varepsilon}
$$

are given by

$$
\begin{aligned}
\left(H^{\varepsilon} u\right)_{11}=\frac{1}{\varepsilon} g_{1}^{\varepsilon} \cdot \partial_{1} u, & \left(H^{\varepsilon} u\right)_{12}=\frac{1}{\delta_{\varepsilon}} g_{1}^{\varepsilon} \cdot \partial_{2} u, & & \left(H^{\varepsilon} u\right)_{13}=g_{1}^{\varepsilon} \cdot \partial_{3} u \\
\left(H^{\varepsilon} u\right)_{21}=\frac{1}{\varepsilon} g_{2}^{\varepsilon} \cdot \partial_{1} u, & \left(H^{\varepsilon} u\right)_{22}=\frac{1}{\delta_{\varepsilon}} g_{2}^{\varepsilon} \cdot \partial_{2} u, & & \left(H^{\varepsilon} u\right)_{23}=g_{2}^{\varepsilon} \cdot \partial_{3} u \\
\left(H^{\varepsilon} u\right)_{31}=\frac{1}{\varepsilon} g_{3}^{\varepsilon} \cdot \partial_{1} u, & \left(H^{\varepsilon} u\right)_{32}=\frac{1}{\delta_{\varepsilon}} g_{3}^{\varepsilon} \cdot \partial_{2} u, & & \left(H^{\varepsilon} u\right)_{33}=g_{3}^{\varepsilon} \cdot \partial_{3} u
\end{aligned}
$$

Likewise,

$$
\left(E^{\varepsilon} u\right)_{i j}:=g_{i}^{\varepsilon} \cdot E^{\varepsilon} u g_{j}^{\varepsilon}=\frac{\left(H^{\varepsilon} u\right)_{i j}+\left(H^{\varepsilon} u\right)_{j i}}{2}
$$

By setting $\mathscr{L}_{\varepsilon}(u):=\hat{\mathscr{L}}_{\varepsilon}(\hat{u}) /\left(\varepsilon \delta_{\varepsilon}\right)$ and $\sqrt{g^{\varepsilon}}:=g_{1}^{\varepsilon} \cdot g_{2}^{\varepsilon} \times g_{3}^{\varepsilon}=1-\left(\delta_{\varepsilon} / \varepsilon\right) x_{2} \kappa$, from (6) we get

$$
\frac{\hat{\mathscr{F}}_{\varepsilon}(\hat{u})}{\varepsilon \delta_{\varepsilon}}=\frac{1}{2} \int_{\Omega} \mathbb{C} E^{\varepsilon} u \cdot E^{\varepsilon} u \sqrt{g^{\varepsilon}} d x-\mathscr{L}_{\varepsilon}(u)=: \mathscr{F}_{\varepsilon}(u),
$$

with $\Omega:=(0, \ell) \times\left(-\frac{h}{2}, \frac{h}{2}\right) \times(0, L)$ and $\mathbb{C}:=\mathbb{C}^{\varepsilon} \circ \chi_{\varepsilon}$. Hereafter we assume that $\mathbb{C}=\mathbb{C}(x)$ does not depend on $\varepsilon$. From (4) it follows that: there exists $c>0$ with

$$
\mathbb{C}(x) E \cdot E \geq c|E|^{2}
$$

for almost every $x$ and for all symmetric matrices $E$.

Let

$$
H_{d n}^{1}\left(\Omega ; \mathbb{R}^{3}\right):=\left\{u \in H^{1}\left(\Omega ; \mathbb{R}^{3}\right): u=0 \text { on } \omega \times\{0\}\right\}
$$

with $\omega:=(0, \ell) \times\left(-\frac{h}{2}, \frac{h}{2}\right)$. Then, by taking the periodicity of $\gamma$ into account, (5) yields that the domain of the energy functional $\mathscr{F}_{\varepsilon}$ is

$$
H_{\# d n}^{1}\left(\Omega ; \mathbb{R}^{3}\right):=\left\{u \in H_{d n}^{1}\left(\Omega ; \mathbb{R}^{3}\right): u(0, \cdot, \cdot)=u(\ell, \cdot, \cdot)\right\} .
$$

\section{Compactness Results}

Throughout the section we consider a sequence of functions $u^{\varepsilon} \in H_{\# d n}^{1}\left(\Omega ; \mathbb{R}^{3}\right)$ such that

$$
\sup _{\varepsilon} \frac{1}{\delta_{\varepsilon}}\left\|E^{\varepsilon} u^{\varepsilon}\right\|_{L^{2}(\Omega)}<+\infty .
$$

Since $H_{\# d n}^{1}\left(\Omega ; \mathbb{R}^{3}\right) \subset H_{d n}^{1}\left(\Omega ; \mathbb{R}^{3}\right)$ it follows that the compactness results proved in [1], for thin walled beams with open cross-sections, hold also in the present context. The following theorem summarizes some results proved in Section 5 of [1].

Theorem 4.1. There exists a sequence $W^{\varepsilon} \in H^{1}\left((0, \ell) \times(0, L) ; \mathbb{R}_{\mathrm{skw}}^{3 \times 3}\right)$ such that $W^{\varepsilon}(0, \cdot)=W^{\varepsilon}(\ell, \cdot)$ and

i) $\left\|H^{\varepsilon} u^{\varepsilon}-W^{\varepsilon}\right\|_{L^{2}(\Omega)} \leq C \delta_{\varepsilon}$,

ii) $\left\|W^{\varepsilon}\right\|_{L^{2}(\Omega)}+\left\|\partial_{3} W^{\varepsilon}\right\|_{L^{2}(\Omega)} \leq C$,

iii) $\left\|\partial_{1} W^{\varepsilon}\right\|_{L^{2}(\Omega)} \leq C \varepsilon$, 
for a suitable $C>0$ and every $\varepsilon$ small enough. Moreover, there exist $W \in$ $H_{d n}^{1}\left((0, L) ; \mathbb{R}_{\mathrm{skw}}^{3 \times 3}\right)$ and $B \in L^{2}\left((0, \ell) \times(0, L) ; \mathbb{R}_{\mathrm{skw}}^{3 \times 3}\right)$ such that, up to a subsequence,

$$
W^{\varepsilon} \rightarrow W \text { in } H^{1}\left((0, \ell) \times(0, L) ; \mathbb{R}^{3 \times 3}\right),
$$$$
\frac{\partial_{1} W^{\varepsilon}}{\varepsilon} \rightarrow B \text { in } L^{2}\left((0, \ell) \times(0, L) ; \mathbb{R}^{3 \times 3}\right),
$$

and

$$
u^{\varepsilon} \rightarrow 0 \text { in } H_{d n}^{1}\left(\Omega ; \mathbb{R}^{3}\right) .
$$

Since the limit of $u^{\varepsilon}$ is equal to zero, the following rescaled components of $u^{\varepsilon}$ were considered in [1]:

$$
\bar{v}^{\varepsilon}:=\frac{u^{\varepsilon}-u_{3}^{\varepsilon} e_{3}}{\delta_{\varepsilon} / \varepsilon}, \quad v_{3}^{\varepsilon}:=\frac{u_{3}^{\varepsilon}}{\delta_{\varepsilon}}
$$

From (19) it follows that

$$
W_{21}^{\varepsilon}=g_{2}^{\varepsilon} \cdot W^{\varepsilon} g_{1}^{\varepsilon} \rightarrow n \cdot W t=: \vartheta \text { in } H^{1}((0, \ell) \times(0, L)),
$$

with $\vartheta \in H_{d n}^{1}(0, L)$. In [1], for thin-walled beams with open cross-section, it was shown that $\vartheta$ represents the rotation of the cross-section around the $x_{3}$-axis. It turns out that for closed cross-sections $\vartheta$ is equal to zero, see Theorem 4.4 below, hence the sequence $\left\{W_{21}^{\varepsilon}\right\}$ is too 'crude' to capture the rotation of the cross-section in the limit. A measure of the rotation suitable for closed cross-sections is instead given by

$$
\Theta^{\varepsilon}:=\frac{-1}{f_{0}^{\ell} \gamma \cdot n d x_{1}} f_{\omega} \frac{g_{1}^{\varepsilon} \cdot u^{\varepsilon}}{\delta_{\varepsilon}} d a
$$

Since $n=e_{3} \wedge t$, the quantity $\int_{0}^{\ell} \gamma \cdot n d x_{1}$ is equal to minus twice the area inside the curve $\gamma$, and hence it is different from zero. The quantities $\Theta^{\varepsilon}$ are in fact averages of the local rotations suitably rescaled at a finer scale, see Theorem 4.2 and Remark 4.3 below.

The following identity, which will be crucial in our analysis,

$$
f_{\omega} \frac{2\left(E^{\varepsilon} u^{\varepsilon}\right) 13}{\delta_{\varepsilon}} d a=\partial_{3} \int_{\omega} \frac{g_{1}^{\varepsilon} \cdot u^{\varepsilon}}{\delta_{\varepsilon}} d a
$$

can be deduced from

$$
f_{\omega} \frac{2\left(E^{\varepsilon} u^{\varepsilon}\right) 13}{\delta_{\varepsilon}} d a=\frac{1}{\varepsilon \delta_{\varepsilon}} f_{\omega} \partial_{1} u_{3}^{\varepsilon} d a+\frac{1}{\delta_{\varepsilon}} f_{\omega} g_{1}^{\varepsilon} \cdot \partial_{3} u^{\varepsilon} d a
$$

after noticing that the first integral on the right-hand side vanishes in the case of a closed cross-section.

Theorem 4.2. There exists $\Theta \in H_{d n}^{1}(0, L)$ such that, up to a subsequence,

$$
\Theta^{\varepsilon} \rightarrow \Theta \quad \text { in } H^{1}(0, L)
$$

and

$$
\frac{\varepsilon}{\delta_{\varepsilon}} \frac{1}{f_{0}^{\ell} \gamma \cdot n d x_{1}} f_{\omega} g_{2}^{\varepsilon} \cdot W^{\varepsilon} g_{1}^{\varepsilon} \gamma \cdot n d a \rightarrow \Theta \quad \text { in } L^{2}(0, L)
$$


Proof. From (18), (24), and since $u^{\varepsilon} \in H_{\# d n}^{1}\left(\Omega ; R^{3}\right)$, we immediately deduce that

$$
\sup _{\varepsilon}\left\|f_{\omega} \frac{g_{1}^{\varepsilon} \cdot u^{\varepsilon}}{\delta_{\varepsilon}} d a\right\|_{H^{1}(0, L)}<+\infty
$$

and hence $\sup _{\varepsilon}\left\|\Theta^{\varepsilon}\right\|_{H^{1}(0, L)}<+\infty$. Thus, statement (25) follows.

Since

$$
f_{\omega} \frac{g_{1}^{\varepsilon} \cdot u^{\varepsilon}}{\delta_{\varepsilon}} d a=\frac{1}{\delta_{\varepsilon}} f_{\omega} u^{\varepsilon} \cdot \partial_{1}\left(\gamma+\frac{\delta_{\varepsilon}}{\varepsilon} x_{2} n\right) d a=-\frac{1}{\delta_{\varepsilon}} f_{\omega} \partial_{1} u^{\varepsilon} \cdot\left(\gamma+\frac{\delta_{\varepsilon}}{\varepsilon} x_{2} n\right) d a,
$$

by means of the identity, see (13),

$$
\frac{1}{\varepsilon} \partial_{1} u^{\varepsilon}=H^{\varepsilon} u^{\varepsilon} g_{1}^{\varepsilon}
$$

we find that

$f_{\omega} \frac{g_{1}^{\varepsilon} \cdot u^{\varepsilon}}{\delta_{\varepsilon}} d a=-\frac{\varepsilon}{\delta_{\varepsilon}} f_{\omega} W^{\varepsilon} g_{1}^{\varepsilon} \cdot\left(\gamma+\frac{\delta_{\varepsilon}}{\varepsilon} x_{2} n\right) d a-\frac{\varepsilon}{\delta_{\varepsilon}} f_{\omega}\left(H^{\varepsilon} u^{\varepsilon}-W^{\varepsilon}\right) g_{1}^{\varepsilon} \cdot\left(\gamma+\frac{\delta_{\varepsilon}}{\varepsilon} x_{2} n\right) d a$.

By using that $\gamma+\frac{\delta_{\varepsilon}}{\varepsilon} x_{2} n$ is orthogonal to $e_{3}$ and that $W^{\varepsilon} g_{1}^{\varepsilon} \cdot g_{1}^{\varepsilon}=0$, since $W^{\varepsilon}$ is skew symmetric, the above equation can be rewritten as

$$
\begin{aligned}
f_{\omega} \frac{g_{1}^{\varepsilon} \cdot u^{\varepsilon}}{\delta_{\varepsilon}} d a=-\frac{\varepsilon}{\delta_{\varepsilon}} f_{\omega} W^{\varepsilon} g_{1}^{\varepsilon} & \cdot g_{2}^{\varepsilon}\left(\gamma \cdot n+\frac{\delta_{\varepsilon}}{\varepsilon} x_{2}\right) d a \\
& -\frac{\varepsilon}{\delta_{\varepsilon}} f_{\omega}\left(H^{\varepsilon} u^{\varepsilon}-W^{\varepsilon}\right) g_{1}^{\varepsilon} \cdot\left(\gamma+\frac{\delta_{\varepsilon}}{\varepsilon} x_{2} n\right) d a .
\end{aligned}
$$

Dividing (27) by $-f_{0}^{\ell} \gamma \cdot n d x_{1}$ and taking the limit, we get

$$
\Theta^{\varepsilon}-\frac{\varepsilon}{\delta_{\varepsilon}} \frac{1}{f_{0}^{\ell} \gamma \cdot n d x_{1}} f_{\omega} g_{2}^{\varepsilon} \cdot W^{\varepsilon} g_{1}^{\varepsilon} \gamma \cdot n d a \rightarrow f_{\omega} W t \cdot n x_{2} d a \quad L^{2}(0, L)
$$

where we have used $i$ ) of Theorem 4.1. Since $W t \cdot n$ is independent of $x_{2}$ it follows that the limit above is equal to zero and hence (26) follows from (25).

Remark 4.3. Theorem 4.2 illustrates the meaning of $\Theta^{\varepsilon}$. It follows from (26) that the field $\Theta$ is the limit of a weighted average of the local infinitesimal rotations around the axis $x_{3}$, which is represented by $W_{21}^{\varepsilon}$, scaled by $\varepsilon / \delta_{\varepsilon}$. In particular, we notice that the definition of the sequence of rotations $\Theta^{\varepsilon}$ does not involve the displacement $u^{\varepsilon}$ only, as in the case of open cross-sections, but also the shape of the cross-section through the factor $\left(\gamma+\frac{\delta_{\varepsilon}}{\varepsilon} x_{2} n\right)$ which appears in the proof, see (27). This is in accordance with the fact that the torsional rigidity of a closed cross-section depends on its shape, a property which is well known to engineers. For instance, a circular cross-section has a torsional rigidity much larger than an ellipsoidal cross-section with the same length of $\gamma$ but with a small ratio between the lengths of the axes.

Theorem 4.4. Let $\vartheta$ be as in (22). Then, $\vartheta=0$ almost everywhere in $(0, L)$.

Proof. By (22) we have that

$$
f_{\omega} W^{\varepsilon} g_{1}^{\varepsilon} \cdot g_{2}^{\varepsilon} \gamma \cdot n d a \rightarrow \vartheta f_{0}^{\ell} \gamma \cdot n d x_{1} \text { in } H^{1}(0, L)
$$


But from (26) we also have that

$$
f_{\omega} W^{\varepsilon} g_{1}^{\varepsilon} \cdot g_{2}^{\varepsilon} \gamma \cdot n d a \rightarrow 0 \text { in } L^{2}(0, L)
$$

and hence

$$
\vartheta f_{0}^{\ell} \gamma \cdot n d x_{1}=0
$$

Recalling that the quantity $\left|\int_{0}^{\ell} \gamma \cdot n d x_{1}\right|$ is equal to twice the area contained in the simple closed curve $\gamma$, then we must have $\vartheta=0$.

To characterize the limit of the sequence of rescaled displacements $v^{\varepsilon}$ we need to assume the slenderness parameter

$$
\mathfrak{s}:=\lim _{\varepsilon \rightarrow 0} \frac{\varepsilon^{2}}{\delta_{\varepsilon}}
$$

to be finite. More precisely, throughout the paper, we shall assume that

$$
\mathfrak{s} \in\{0,1\} .
$$

Taking into account that $\vartheta=0$, we may rewrite Theorem 5.7 and Theorem 5.8 of [1] as follows.

Theorem 4.5. Let

$$
\gamma_{G}:=\int_{0}^{\ell} \gamma\left(x_{1}\right) d x_{1}
$$

There exist

$$
\bar{m} \in H_{d n}^{2}\left(0, L ; \mathbb{R}^{3}\right):=\left\{z \in H^{2}\left(0, L ; \mathbb{R}^{3}\right): z(0)=\partial_{3} z(0)=0\right\}
$$

and $m_{3} \in H_{d n}^{1}(0, L)$ such that, up to a subsequence, we have

i) $\bar{v}^{\varepsilon} \rightarrow \bar{m}$ in $H_{d n}^{1}\left(\Omega ; \mathbb{R}^{3}\right)$, with $\bar{m}_{3}=0$,

ii) $v_{3}^{\varepsilon} \rightarrow v_{3}:=m_{3}-\partial_{3} \bar{m} \cdot\left(\gamma-\gamma_{G}\right)$ in $H_{d n}^{1}(\Omega)$.

We conclude the section by studying the limit behaviour of the rescaled strains. From (18) we deduce that there exists $E \in L^{2}\left(\Omega ; \mathbb{R}_{\text {sym }}^{3 \times 3}\right)$ such that, up to a subsequence,

$$
\frac{E^{\varepsilon} u^{\varepsilon}}{\delta_{\varepsilon}} \rightarrow E \text { in } L^{2}\left(\Omega ; \mathbb{R}^{3 \times 3}\right)
$$

We recall from [1] that $E_{13}:=t \cdot E e_{3}$ satisfies

$$
\partial_{2} E_{13}=0
$$

and

$$
E_{11}:=t \cdot E t=x_{2} \eta_{3}+\eta_{1},
$$

where $\eta_{1} \in L^{2}(\Omega), \eta_{3}=t \cdot B n$, and $\partial_{2} \eta_{1}=\partial_{2} \eta_{3}=0$, with $B$ defined in (20).

The next lemma integrates these results for closed cross-sections.

Lemma 4.6. The following representations hold:

i) $E_{33}:=e_{3} \cdot E e_{3}=\partial_{3} v_{3}$, 
ii) there exists $\eta_{2} \in L^{2}(\Omega)$ with

$$
\partial_{2} \eta_{2}=0 \quad \text { and } \quad \int_{0}^{\ell} \eta_{2} d x_{1}=0 \text { a.e. in }\left(-\frac{h}{2}, \frac{h}{2}\right) \times(0, L)
$$

such that

$$
E_{13}=\eta_{2}-\frac{1}{2} \partial_{3} \Theta \gamma \cdot n,
$$

iii) there exist $\eta_{1} \in L^{2}(\Omega), \eta_{3} \in H^{1}(\Omega)$ with

$$
\begin{gathered}
\partial_{2} \eta_{1}=\partial_{2} \eta_{3}=0 \\
\int_{0}^{\ell} \eta_{3} d x_{1}=0 \text { a.e. in }\left(-\frac{h}{2}, \frac{h}{2}\right) \times(0, L), \\
\int_{0}^{\ell} t \int_{0}^{s} \eta_{3} d x_{1} d s=0 \text { a.e. in }(0, L),
\end{gathered}
$$

such that

$$
E_{11}=x_{2} \eta_{3}+\eta_{1}
$$

Proof. $i$ ) follows by passing to the limit in

$$
\frac{\left(E^{\varepsilon} u^{\varepsilon}\right)_{33}}{\delta_{\varepsilon}}=\frac{g_{3}^{\varepsilon} \cdot \partial_{3} u^{\varepsilon}}{\delta_{\varepsilon}}=\partial_{3} v_{3}^{\varepsilon}
$$

and by applying $i$ i) of Theorem 4.5.

As to point $i i)$, from $(23),(24),(28)$ and $(25)$, we deduce that

$$
2 f_{\omega} E_{13} d x_{1}=-\partial_{3} \Theta f_{0}^{\ell} \gamma \cdot n d x_{1} \text {. }
$$

Since, by (29), $\partial_{2} E_{13}=0$ we can write

(34) $E_{13}=\eta_{2}-\frac{1}{2} \partial_{3} \Theta \gamma \cdot n$, with $\eta_{2} \in L^{2}(\Omega), \partial_{2} \eta_{2}=0 \quad$ and $\quad \int_{0}^{\ell} \eta_{2} d x_{1}=0$, which is $i i)$.

We now prove $i i i)$. By (20) it follows that

$$
\lim _{\varepsilon \rightarrow 0} \int_{0}^{L} \int_{0}^{\ell} \frac{\partial_{1} W^{\varepsilon}}{\varepsilon} \cdot t \otimes n \psi\left(x_{3}\right) d x_{1} d x_{3}=\int_{0}^{L} \int_{0}^{\ell} B \cdot t \otimes n \psi\left(x_{3}\right) d x_{1} d x_{3}
$$

for all $\psi \in L^{2}(0, L)$. On the other hand, since $W^{\varepsilon}$ is skew symmetric, the following identity holds

$$
\partial_{1} W^{\varepsilon} \cdot t \otimes n=\partial_{1}\left(W^{\varepsilon} \cdot t \otimes n\right)
$$

and hence

$$
\begin{aligned}
\lim _{\varepsilon \rightarrow 0} \int_{0}^{L} \int_{0}^{\ell} \frac{\partial_{1} W^{\varepsilon}}{\varepsilon} \cdot t \otimes n \psi\left(x_{3}\right) d x_{1} d x_{3} \\
\quad=\lim _{\varepsilon \rightarrow 0} \int_{0}^{L} \int_{0}^{\ell} \frac{\partial_{1}\left(W^{\varepsilon} \cdot t \otimes n\right)}{\varepsilon} \psi\left(x_{3}\right) d x_{1} d x_{3}=0
\end{aligned}
$$

because $W^{\varepsilon}(0, \cdot)=W^{\varepsilon}(\ell, \cdot)$. Thus, from (35) we have

$$
\int_{0}^{L} \psi\left(x_{3}\right) \int_{0}^{\ell} B \cdot t \otimes n d x_{1} d x_{3}=0
$$


which implies

$$
\int_{0}^{\ell} B \cdot t \otimes n d x_{1}=0
$$

From this identity and (30) it follows that $\int_{0}^{\ell} \eta_{3} d x_{1}=0$.

Finally, in order to prove that $\int_{0}^{\ell} t \int_{0}^{s} \eta_{3} d x_{1} d s=0$, we set

$$
W_{t n}^{\varepsilon}:=t \cdot W^{\varepsilon} n, \quad\left(H^{\varepsilon} u^{\varepsilon}\right)_{t n}:=t \cdot H^{\varepsilon} u^{\varepsilon} n,
$$

and remark that, as before, we have

$$
\partial_{1} W_{t n}^{\varepsilon}=\partial_{1}\left(t \cdot W^{\varepsilon} n\right)=t \cdot\left(\partial_{1} W^{\varepsilon}\right) n
$$

because $W^{\varepsilon}$ is skew-symmetric. Hence, by (20) and (30) we get

$$
\frac{\partial_{1} W_{t n}^{\varepsilon}}{\varepsilon} \rightarrow t \cdot B n=\eta_{3}
$$

in $L^{2}(\Omega)$. By a partial Poincaré inequality, see for instance [7] Theorem 4.1, there exists a positive constant $C$ such that

$$
\left\|\frac{W_{t n}^{\varepsilon}}{\varepsilon}-f_{0}^{\ell} \frac{W_{t n}^{\varepsilon}}{\varepsilon} d x_{1}\right\|_{L^{2}(\Omega)} \leq C\left\|\frac{\partial_{1} W_{t n}^{\varepsilon}}{\varepsilon}\right\|_{L^{2}(\Omega)}
$$

for any $\varepsilon>0$. This, together with (36), implies

$$
\frac{W_{t n}^{\varepsilon}}{\varepsilon}-f_{0}^{\ell} \frac{W_{t n}^{\varepsilon}}{\varepsilon} d x_{1} \rightarrow \int_{0}^{x_{1}} \eta_{3} d x_{1}-f_{0}^{\ell} \int_{0}^{s} \eta_{3} d x_{1} d s
$$

in $L^{2}(\Omega)$. By $i$ ) of Theorem 4.1 and using the fact that $\delta_{\varepsilon} / \varepsilon \rightarrow 0$, we deduce that (37) still holds true if $W^{\varepsilon}$ is replaced by $H^{\varepsilon} u^{\varepsilon}$. Then, multiplying by $g_{2}^{\varepsilon}=n$ on both sides we have that

$$
\frac{\left(H^{\varepsilon} u^{\varepsilon}\right)_{t n}}{\varepsilon} g_{2}^{\varepsilon}-f_{0}^{\ell} \frac{\left(H^{\varepsilon} u^{\varepsilon}\right)_{t n}}{\varepsilon} d x_{1} g_{2}^{\varepsilon} \rightarrow n \int_{0}^{x_{1}} \eta_{3} d x_{1}-n f_{0}^{\ell} \int_{0}^{s} \eta_{3} d x_{1} d s
$$

in $L^{2}(\Omega)$. By integrating with respect to $x_{1}$ on the interval $(0, \ell)$ and by taking into account that

it follows that

$$
\int_{0}^{\ell} g_{2}^{\varepsilon} d x_{1}=\int_{0}^{\ell} n d x_{1}=0
$$

$$
\int_{0}^{\ell} \frac{\left(H^{\varepsilon} u^{\varepsilon}\right)_{t n}}{\varepsilon} g_{2}^{\varepsilon} d x_{1} \rightarrow \int_{0}^{\ell} n \int_{0}^{s} \eta_{3} d x_{1} d s
$$

in $L^{2}((-h / 2, h / 2) \times(0, L))$. Therefore, from

$$
\left(H^{\varepsilon} u^{\varepsilon}\right)_{12}=g_{1}^{\varepsilon} \cdot H^{\varepsilon} u^{\varepsilon} g_{2}^{\varepsilon}=\left(1-\frac{\delta_{\varepsilon}}{\varepsilon} x_{2} \kappa\right) t \cdot H^{\varepsilon} u^{\varepsilon} n=\left(1-\frac{\delta_{\varepsilon}}{\varepsilon} x_{2} \kappa\right)\left(H^{\varepsilon} u^{\varepsilon}\right)_{t n},
$$

we get

$$
\int_{0}^{\ell} \frac{\left(H^{\varepsilon} u^{\varepsilon}\right) 12}{\varepsilon} g_{\varepsilon}^{2} d x_{1} \rightarrow \int_{0}^{\ell} n \int_{0}^{s} \eta_{3} d x_{1} d s
$$

in $L^{2}((-h / 2, h / 2) \times(0, L))$.

Let us write $u^{\varepsilon}$ in the following form

$$
u^{\varepsilon}=\varepsilon \delta_{\varepsilon} x_{2} \int_{0}^{x_{1}} \eta_{3} d x_{1} t+\varepsilon \delta_{\varepsilon} \mathfrak{r}^{\varepsilon},
$$

where the "remainder" $\mathfrak{r}^{\varepsilon}$ is defined by the equality above and thus satisfies the periodicity conditions: $\mathfrak{r}_{\mid x_{1}=0}^{\varepsilon}=\mathfrak{r}_{\mid x_{1}=\ell}^{\varepsilon}=0$. 
Since

$$
\frac{\left(E^{\varepsilon} u^{\varepsilon}\right)_{11}}{\delta_{\varepsilon}}=\frac{1}{\delta_{\varepsilon}}\left(g_{1}^{\varepsilon} \cdot \frac{\partial_{1} u^{\varepsilon}}{\varepsilon}\right)=x_{2} \eta_{3}\left(1-\frac{\delta_{\varepsilon}}{\varepsilon} x_{2} \kappa\right)+g_{1}^{\varepsilon} \cdot \partial_{1} \mathfrak{r}^{\varepsilon},
$$

by taking the limit as $\varepsilon \rightarrow 0$ and recalling that $E_{11}=x_{2} \eta_{3}+\eta_{1}$ we obtain

$$
g_{1}^{\varepsilon} \cdot \partial_{1} \mathfrak{r}^{\varepsilon} \rightarrow \eta_{1}
$$

in $L^{2}(\Omega)$. Furthermore, by observing that

$\frac{\delta_{\varepsilon}}{\varepsilon} \frac{2\left(E^{\varepsilon} u^{\varepsilon}\right)_{12}}{\delta_{\varepsilon}}=\left(1-\frac{\delta_{\varepsilon}}{\varepsilon} x_{2} \kappa\right) \int_{0}^{x_{1}} \eta_{3} d x_{1}+g_{1}^{\varepsilon} \cdot \partial_{2} \mathfrak{r}^{\varepsilon}+\frac{\delta_{\varepsilon}}{\varepsilon} \kappa x_{2} \int_{0}^{x_{1}} \eta_{3} d x_{1}+\frac{\delta_{\varepsilon}}{\varepsilon} g_{2}^{\varepsilon} \cdot \partial_{1} \mathfrak{r}^{\varepsilon}$ and passing to the limit we get, thanks to (18), that

$$
g_{1}^{\varepsilon} \cdot \partial_{2} \mathfrak{r}^{\varepsilon}+\frac{\delta_{\varepsilon}}{\varepsilon} g_{2}^{\varepsilon} \cdot \partial_{1} \mathfrak{r}^{\varepsilon} \rightarrow-\int_{0}^{x_{1}} \eta_{3} d x_{1}
$$

in $L^{2}(\Omega)$. Since

$$
\frac{\left(H^{\varepsilon} u^{\varepsilon}\right)_{12}}{\varepsilon}=\frac{1}{\varepsilon} g_{1}^{\varepsilon} \cdot \frac{\partial_{2} u^{\varepsilon}}{\delta_{\varepsilon}}=\left(1-\frac{\delta_{\varepsilon}}{\varepsilon} x_{2} \kappa\right) \int_{0}^{x_{1}} \eta_{3} d x_{1}+\partial_{2} \mathfrak{r}^{\varepsilon} \cdot g_{1}^{\varepsilon},
$$

if we multiply by $g_{\varepsilon}^{2}$, integrate, pass to the limit, and recall (38), we obtain that

$$
\int_{0}^{\ell} g_{1}^{\varepsilon} \cdot \partial_{2} \mathfrak{r}^{\varepsilon} g_{\varepsilon}^{2} d x_{1} \rightarrow 0
$$

in $L^{2}((-h / 2, h / 2) \times(0, L))$.

From the periodicity of $\mathfrak{r}^{\varepsilon}$ the following identity holds

$$
0=\frac{\delta_{\varepsilon}}{\varepsilon} \int_{0}^{\ell} \partial_{1} \mathfrak{r}^{\varepsilon} d x_{1} .
$$

By representing $\partial_{1} \mathfrak{r}^{\varepsilon}$ in components with respect to the basis $\left(g_{\varepsilon}^{1}, g_{\varepsilon}^{2}, e_{3}\right)$ and adding and subtracting the quantity $\left(\partial_{2} \mathfrak{r}^{\varepsilon} \cdot g_{1}^{\varepsilon}\right) g_{\varepsilon}^{2}$, we get

$$
\begin{aligned}
0= & \frac{\delta_{\varepsilon}}{\varepsilon} \int_{0}^{\ell} \partial_{1} \mathfrak{r}^{\varepsilon} \cdot g_{1}^{\varepsilon} g_{\varepsilon}^{1} d x_{1}+\int_{0}^{\ell} \frac{\delta_{\varepsilon}}{\varepsilon} \partial_{1} \mathfrak{r}^{\varepsilon} \cdot g_{2}^{\varepsilon} g_{\varepsilon}^{2}+\partial_{2} \mathfrak{r}^{\varepsilon} \cdot g_{1}^{\varepsilon} g_{\varepsilon}^{2} d x_{1} \\
& -\int_{0}^{\ell} \partial_{2} \mathfrak{r}^{\varepsilon} \cdot g_{1}^{\varepsilon} g_{\varepsilon}^{2} d x_{1}+\frac{\delta_{\varepsilon}}{\varepsilon} \int_{0}^{\ell} \partial_{1} \mathfrak{r}^{\varepsilon} \cdot e_{3} e_{3} d x_{1} .
\end{aligned}
$$

Only the second integral in the sum above has a non trivial limit. Indeed, the first tends to zero because of (39) and the fact that $\delta_{\varepsilon} / \varepsilon \rightarrow 0$, the third vanishes by (41) and the last one is null by the periodicity conditions. Thus, taking the limit as $\varepsilon \rightarrow 0$ and recalling (40), we obtain

$$
0=\int_{0}^{\ell} n \int_{0}^{s} \eta_{3} d x_{1} d s .
$$

The claimed result follows by observing that $n=e_{3} \wedge t$.

Remark 4.7. It is worth noticing that part $i i$ ) of Lemma 4.6 can be written in an equivalent form. Let us set

$$
\varphi:=2 \int_{0}^{x_{1}} \eta_{2} d x_{1}+c,
$$

with $c$ dependent on $x_{3}$ only. Then, part $\left.i i\right)$ can be restated as 
ii) there exists $\varphi \in H^{1}\left((0, \ell) ; L^{2}(0, L)\right)$, with $\varphi\left(0, x_{3}\right)=\varphi\left(\ell, x_{3}\right)$, such that

$$
2 E_{13}=\partial_{1} \varphi-\partial_{3} \Theta \gamma \cdot n \text {. }
$$

This equation evokes the classical formula for the warping function in Vlasov's theory, as better explained in Remark 8.1.

\section{REDUCED ENERGY FOR CLOSED CROSS-SECTIONS}

Here we introduce two reduced energy densities that will be used to compute the $\Gamma$-limit. The first of them is obtained by minimizing the energy with respect to the strain components that have not been characterized by the analysis of Section 4 . The second, by a minimization with respect to the functions $\eta_{i}$ introduced in Lemma 4.6. While in the former case the analysis is algebraic and coincides with that done in [1] for the open cross-sections, in the latter it is not simply algebraic because of the integral constraints on $\eta_{i}$.

Let

$$
f(x, M):=\frac{1}{2} \mathbb{C}(x) M \cdot M,
$$

be the elastic energy density of the body, with $M$ a symmetric $3 \times 3$ matrix and $\mathbb{C}$ the elasticity tensor introduced in Section 2. We define

$$
\begin{aligned}
f_{0}\left(x, M_{11}, M_{13}, M_{33}\right):=\min _{D_{i j}} f(x, & M_{11} t\left(x_{1}\right) \odot t\left(x_{1}\right)+2 D_{12} t\left(x_{1}\right) \odot n\left(x_{1}\right) \\
& +2 M_{13} t\left(x_{1}\right) \odot e_{3}+D_{22} n\left(x_{1}\right) \odot n\left(x_{1}\right) \\
& \left.+2 D_{23} n\left(x_{1}\right) \odot e_{3}+M_{33} e_{3} \odot e_{3}\right),
\end{aligned}
$$

the function obtained from $f$ by keeping fixed the components that have been partially characterized in Section 4 and by minimizing over the remaining components.

Let the space of symmetric tensors be decomposed in the direct sum of the subspaces

$$
\mathcal{S}\left(x_{1}\right):=\operatorname{span}\left\{t\left(x_{1}\right) \odot n\left(x_{1}\right), n\left(x_{1}\right) \odot n\left(x_{1}\right), n\left(x_{1}\right) \odot e_{3}\right\}
$$

and

$$
\mathcal{S}^{\perp}\left(x_{1}\right):=\operatorname{span}\left\{t\left(x_{1}\right) \odot t\left(x_{1}\right), t\left(x_{1}\right) \odot e_{3}, e_{3} \odot e_{3}\right\},
$$

so that any tensor $M \in \mathbb{R}_{\mathrm{sym}}^{3 \times 3}$ can be uniquely written as

$$
M=M^{\mathcal{S}}+M^{\perp},
$$

with

$$
M^{\mathcal{S}}:=2(t \cdot M n) t \odot n+(n \cdot M n) n \odot n+2\left(n \cdot M e_{3}\right) n \odot e_{3} \in \mathcal{S}
$$

and

$$
M^{\perp}:=M_{11} t \odot t+2 M_{13} t \odot e_{3}+M_{33} e_{3} \odot e_{3} \in \mathcal{S}^{\perp},
$$

where

$$
M_{11}:=t \cdot M t, \quad M_{13}:=t \cdot M e_{3}, \quad M_{33}:=e_{3} \cdot M e_{3} .
$$

The decomposition of $E$, as given by (28), is such that $E^{\perp}$ contains the components of $E$ that have been partially characterized in Lemma 4.6, while $E^{\mathcal{S}}$ contains the remaining ones.

As shown in Section 6 of [1], the minimization problem (44) defines a map

$$
\mathbb{E}_{0}: \mathcal{S}^{\perp} \mapsto \mathcal{S} \oplus \mathcal{S}^{\perp}
$$


that sends every $M^{\perp} \in \mathcal{S}^{\perp}$ into the corresponding "full" strain $\left(M_{0}^{\mathcal{S}}+M^{\perp}\right)$ that minimizes $f$ :

$$
f_{0}\left(x, M_{11}, M_{13}, M_{33}\right)=f\left(x, \mathbb{E}_{0} M^{\perp}\right) .
$$

Hereafter, we find it convenient to use also the notation

$$
f_{0}\left(x, M^{\perp}\right):=f_{0}\left(x, M_{11}, M_{13}, M_{33}\right),
$$

so to have the more compact relation

$$
f_{0}\left(x, M^{\perp}\right)=f\left(x, \mathbb{E}_{0} M^{\perp}\right) .
$$

Other properties of $\mathbb{E}_{0}$ are proved in Lemma 6.1 of [1]. In particular, it is shown that $M \in \mathbb{E}_{0} \mathcal{S}^{\perp}$ if and only if

$$
\begin{aligned}
& \mathbb{C} M \cdot t \odot n=0, \\
& \mathbb{C} M \cdot n \odot n=0, \\
& \mathbb{C} M \cdot n \odot e_{3}=0 .
\end{aligned}
$$

It is easy to see that these conditions are equivalent to

$$
\mathbb{C} M \cdot M^{\perp}=\mathbb{C} M \cdot \mathbb{E}_{0} M^{\perp} \quad \forall M^{\perp} \in \mathcal{S}^{\perp} .
$$

It turns out that $f_{0}\left(x, M^{\perp}\right)$ takes the form

$$
\begin{aligned}
f_{0}\left(x, M_{11}, M_{13}, M_{33}\right) & =\frac{1}{2} \mathbb{E}_{0}^{T} \mathbb{C E}_{0} M^{\perp} \cdot M^{\perp} \\
= & \frac{1}{2}\left(\begin{array}{lll}
\mathbb{C}_{11}(x) & \mathbb{C}_{12}(x) & \mathbb{C}_{13}(x) \\
\mathbb{C}_{12}(x) & \mathbb{C}_{22}(x) & \mathbb{C}_{23}(x) \\
\mathbb{C}_{13}(x) & \mathbb{C}_{23}(x) & \mathbb{C}_{33}(x)
\end{array}\right)\left(\begin{array}{l}
M_{11} \\
M_{13} \\
M_{33}
\end{array}\right) \cdot\left(\begin{array}{l}
M_{11} \\
M_{13} \\
M_{33}
\end{array}\right),
\end{aligned}
$$

where the reduced elasticity constants $\mathbb{C}_{i j}(x)$ can be computed in terms of the original constants $\mathbb{C}_{i j h k}(x)$, see equation (60) of [1].

Let us now consider the optimization with respect to the components of $E^{\perp}$. For any given pair of functions $\left(\partial_{3} \Theta, \partial_{3} v_{3}\right)$ with the features described by Theorems 4.2 and 4.5 , let us consider the minimum problem

$$
\min _{\eta_{1}, \eta_{2}, \eta_{3}} \int_{\Omega} f_{0}\left(x, x_{2} \eta_{3}+\eta_{1}, \eta_{2}-\frac{1}{2} \partial_{3} \Theta \gamma \cdot n, \partial_{3} v_{3}\right) d x
$$

where $\eta_{i}$ are as in Lemma 4.6.

The minimization is no longer algebraic, as was the case for the open crosssections, but it turns out that a minimizing triad is uniquely determined and depends linearly upon the pair $\left(\partial_{3} \Theta, \partial_{3} v_{3}\right)$. A constructive characterization of the minimizing triad is provided in the next section.

Let $\mathscr{E}^{\perp}$ be the set of all tensor fields

$$
\left(x_{2} \eta_{3}+\eta_{1}\right) t \odot t+\left(2 \eta_{2}-a \gamma \cdot n\right) t \odot e_{3}+b e_{3} \odot e_{3},
$$

with $a \in L^{2}(0, L) ; b$ and $\eta_{i} \in L^{2}((0, \ell) \times(0, L)), i=1,2,3 ; \eta_{2}, \eta_{3}$ satisfying the constraints

$$
\int_{0}^{\ell} \eta_{2} d x_{1}=\int_{0}^{\ell} \eta_{3} d x_{1}=\int_{0}^{\ell} t \int_{0}^{s} \eta_{3} d x_{1} d s=0 .
$$

It can be checked that any tensor field in $\mathscr{E} \perp$ admits a unique representation in terms of $a, b, \eta_{1}, \eta_{2}, \eta_{3}$.

Let also $\mathscr{E}^{K}$ be the set of all tensor fields

$$
E^{K}(a, b):=-\gamma \cdot n a t \odot e_{3}+b e_{3} \odot e_{3},
$$


with $a \in L^{2}(0, L)$ and $b \in L^{2}((0, \ell) \times(0, L))$.

Let $\mathbb{E}_{00}: \mathscr{E}^{K} \rightarrow \mathscr{E}^{\perp}$ be the map

$$
E^{K}(a, b) \mapsto\left(x_{2} \eta_{3}^{\mathrm{opt}}+\eta_{1}^{\mathrm{opt}}\right) t \odot t+\left(2 \eta_{2}^{\mathrm{opt}}-\gamma \cdot n a\right) t \odot e_{3}+b e_{3} \odot e_{3}
$$

where $\left(\eta_{1}^{\mathrm{opt}}, \eta_{2}^{\mathrm{opt}}, \eta_{3}^{\mathrm{opt}}\right)$ denotes the triad that solves

$$
\min _{\eta_{1}, \eta_{2}, \eta_{3}} \int_{\Omega} f_{0}\left(x, x_{2} \eta_{3}+\eta_{1}, \eta_{2}-\frac{1}{2} a \gamma \cdot n, b\right) d x,
$$

where $\eta_{i}$ are as in Lemma 4.6. It is worth to notice that this minimization problem is well posed since the integral functional is strictly convex, weakly coercive in $L^{2}$, and the constraints are linear. Furthermore, it follows from the analysis in Section 7 that the $\eta_{i}^{\mathrm{opt}}$ are measurable, so that the function $f_{0}\left(x, x_{2} \eta_{3}^{\mathrm{opt}}+\eta_{1}^{\mathrm{opt}}, \eta_{2}^{\mathrm{opt}}-\frac{1}{2} a \gamma \cdot n, b\right)$ is also measurable, see also Remark 6.4 of [1].

The next lemma gives a characterization of the image of $\mathbb{E}_{00}$.

Lemma 5.1. $M \in \mathscr{E}^{\perp}$ is a solution of

$$
\int_{\Omega} \mathbb{C E}_{0} M \cdot\left(\left(x_{2} \varphi_{3}+\varphi_{1}\right) t \odot t+\varphi_{2} t \odot e_{3}\right) d x=0
$$

for all $\varphi_{i} \in L^{2}((0, \ell) \times(0, L)), i=1,2,3$, with $\varphi_{2}, \varphi_{3}$ satisfying the constraints

$$
\int_{0}^{\ell} \varphi_{2} d x_{1}=\int_{0}^{\ell} \varphi_{3} d x_{1}=\int_{0}^{\ell} t \int_{0}^{s} \varphi_{3} d x_{1} d s=0 \text { a.e. in }(0, L),
$$

if and only if

$$
M=\mathbb{E}_{00} E^{K}(a, b)
$$

for some $a \in L^{2}(0, L)$ and $b \in L^{2}((0, \ell) \times(0, L))$.

Proof. Let $M \in \mathscr{E}^{\perp}$. Then, it admits the representation (50) for some appropriate $\eta_{i}, a$, and $b$. It follows that $\eta_{i}=\eta_{i}^{\text {opt }}$, i.e., $M=\mathbb{E}_{00} E^{K}(a, b)$, if and only if $M$ is a solution of the Euler-Lagrange equation for the minimization problem (54) which, using (48), may be written as

$$
\int_{\Omega} \mathbb{E}_{0}^{T} \mathbb{C E}_{0} M \cdot\left(\left(x_{2} \varphi_{3}+\varphi_{1}\right) t \odot t+\varphi_{2} t \odot e_{3}\right) d x=0
$$

for all $\varphi_{i} \in L^{2}((0, \ell) \times(0, L)), i=1,2,3$, with $\varphi_{2}, \varphi_{3}$ satisfying the constraints (55). The $\mathbb{E}_{0}^{T}$ appearing in the equation above can be neglected thanks to (47).

By applying the lemma we deduce that

$$
\int_{\Omega} \mathbb{C E}_{0} \mathbb{E}_{00} E^{K}(a, b) \cdot E^{K}(c, d) d x=\int_{\Omega} \mathbb{C E}_{0} \mathbb{E}_{00} E^{K}(a, b) \cdot \mathbb{E}_{0} \mathbb{E}_{00} E^{K}(c, d) d x
$$

for every $a, c \in L^{2}(0, L)$ and $b, d \in L^{2}((0, \ell) \times(0, L))$. Indeed it suffices to notice that

and use (47).

$$
\int_{\Omega} \mathbb{C E}_{0} \mathbb{E}_{00} E^{K}(a, b) \cdot\left(E^{K}(c, d)-\mathbb{E}_{00} E^{K}(c, d)\right) d x=0
$$

We set $f_{00}: \Omega \times L^{2}(0, L) \times L^{2}((0, \ell) \times(0, L)) \rightarrow L^{1}(\Omega)$ defined by

$$
f_{00}(x, a, b):=f_{0}\left(x, \mathbb{E}_{00} E^{K}(a, b)\right)=f\left(x, \mathbb{E}_{0} \mathbb{E}_{00} E^{K}(a, b)\right),
$$

or, equivalently,

$$
f_{00}(x, a, b):=f_{0}\left(x, x_{2} \eta_{3}^{\mathrm{opt}}+\eta_{1}^{\mathrm{opt}}, \eta_{2}^{\mathrm{opt}}-\frac{1}{2} a \gamma \cdot n, b\right) .
$$


We close the section by noticing that by the definitions of $f_{0}$ and $f_{00}$ we have that

$$
f(x, M) \geq f_{0}\left(x, M^{\perp}\right)
$$

holds for every symmetric matrix $M$, while

$$
\int_{\Omega} f_{0}(x, M) d x \geq \int_{\Omega} f_{00}(x, a, b) d x
$$

holds for every $M \in \mathscr{E}^{\perp}$ with the representation given in (50).

\section{6. $\Gamma$-LIMIT FOR CLOSED CROSS-SECTIONS}

Let $J_{\varepsilon}: H^{1}\left(\Omega ; \mathbb{R}^{3}\right) \rightarrow \mathbb{R} \cup\{+\infty\}$ be defined by

$$
J_{\varepsilon}(u)= \begin{cases}\frac{1}{2} \int_{\Omega} \mathbb{C} E^{\varepsilon} u \cdot E^{\varepsilon} u \sqrt{g^{\varepsilon}} d x & \text { if } u \in H_{\# d n}^{1}\left(\Omega ; \mathbb{R}^{3}\right), \\ +\infty & \text { if } u \in H^{1}\left(\Omega ; \mathbb{R}^{3}\right) \backslash H_{\# d n}^{1}\left(\Omega ; \mathbb{R}^{3}\right) .\end{cases}
$$

In this section we characterize the $\Gamma$-limit of the sequence of functionals $J_{\varepsilon} / \delta_{\varepsilon}^{2}$ in an appropriate topology. In order to define the limit functional we set (62)

$$
\begin{array}{r}
\mathcal{A}_{\#}:=\left\{(\Theta, v) \in H_{d n}^{1}(0, L) \times H^{1}\left(\Omega ; \mathbb{R}^{3}\right): \exists \bar{m} \in H_{d n}^{2}\left(0, L ; \mathbb{R}^{3}\right), \exists m_{3} \in H_{d n}^{1}(0, L)\right. \\
\text { such that } \left.v=\bar{m}+v_{3} e_{3} \text { and } v_{3}=m_{3}-\partial_{3} \bar{m} \cdot\left(\gamma-\gamma_{G}\right)\right\} .
\end{array}
$$

The $\Gamma$-limit will be the functional $J_{\# 0}: H^{1}(0, L) \times H^{1}\left(\Omega ; \mathbb{R}^{3}\right) \rightarrow \mathbb{R} \cup\{+\infty\}$ defined by

$$
J_{\# 0}(\Theta, v)= \begin{cases}\int_{\Omega} f_{00}\left(x, \partial_{3} \Theta, \partial_{3} v_{3}\right) d x & \text { if }(\Theta, v) \in \mathcal{A}_{\#}, \\ +\infty & \text { otherwise }\end{cases}
$$

with $f_{00}$ as in (57).

We start by proving the liminf inequality.

Theorem 6.1 (Liminf inequality). For every sequence $\left\{u^{\varepsilon}\right\} \subset H^{1}\left(\Omega ; \mathbb{R}^{3}\right)$ and every $(\Theta, v) \in H^{1}(0, L) \times H^{1}\left(\Omega ; \mathbb{R}^{3}\right)$ such that

$$
\bar{v}^{\varepsilon}+v_{3}^{\varepsilon} e_{3} \rightarrow v \text { in } H^{1}\left(\Omega ; \mathbb{R}^{3}\right)
$$

and

$$
\frac{-1}{f_{0}^{\ell} \gamma \cdot n d x_{1}} f_{\omega} \frac{g_{1}^{\varepsilon} \cdot u^{\varepsilon}}{\delta_{\varepsilon}} d a \rightarrow \Theta \text { in } H^{1}(0, L)
$$

with $\bar{v}^{\varepsilon}:=\frac{u^{\varepsilon}-u_{3}^{\varepsilon} e_{3}}{\delta_{\varepsilon} / \varepsilon}$ and $v_{3}^{\varepsilon}:=\frac{u_{3}^{\varepsilon}}{\delta_{\varepsilon}}$, we have

$$
\liminf _{\varepsilon \rightarrow 0} \frac{J_{\varepsilon}\left(u^{\varepsilon}\right)}{\delta_{\varepsilon}^{2}} \geq J_{\# 0}(\Theta, v) .
$$

Proof. Without loss of generality we may assume that

$$
\liminf _{\varepsilon \rightarrow 0} \frac{J_{\varepsilon}\left(u^{\varepsilon}\right)}{\delta_{\varepsilon}^{2}}=\lim _{\varepsilon} \frac{J_{\varepsilon}\left(u^{\varepsilon}\right)}{\delta_{\varepsilon}^{2}}<+\infty
$$

since otherwise the claim is trivially satisfied. By the coercivity assumption (17) it follows that the sequence $\left\{u^{\varepsilon}\right\}$ satisfies (18) and hence all theorems contained 
in Sections 4 hold true. In particular, by Theorems 4.2 and 4.5 we have that $(\Theta, v) \in \mathcal{A}_{\#}$. From the fact that $\frac{E^{\varepsilon} u^{\varepsilon}}{\delta_{\varepsilon}} \rightarrow E$ in $L^{2}$ and the convexity of $f$ we find

$$
\begin{aligned}
\liminf _{\varepsilon \rightarrow 0} \frac{J_{\varepsilon}\left(u^{\varepsilon}\right)}{\delta_{\varepsilon}^{2}} & =\liminf _{\varepsilon \rightarrow 0} \int_{\Omega} f\left(x, \frac{E^{\varepsilon} u^{\varepsilon}}{\delta_{\varepsilon}}\right) \sqrt{g^{\varepsilon}} d x \geq \int_{\Omega} f(x, E) d x \\
& \geq \int_{\Omega} f_{0}\left(x, E_{11}, E_{13}, E_{33}\right) d x \\
& \geq \int_{\Omega} f_{00}\left(x, \partial_{3} \Theta, \partial_{3} v_{3}\right) d x=J_{\# 0}(\Theta, v)
\end{aligned}
$$

where the last two inequalities follow from (59), (60) and Lemma 4.6.

We now prove the existence of a recovery sequence.

Theorem 6.2 (Recovery sequence). For every $(\Theta, v) \in H^{1}(0, L) \times H^{1}\left(\Omega ; \mathbb{R}^{3}\right)$ there exists a sequence $\left\{u^{\varepsilon}\right\} \subset H^{1}\left(\Omega ; \mathbb{R}^{3}\right)$ such that

$$
\begin{gathered}
\bar{v}^{\varepsilon}+v_{3}^{\varepsilon} e_{3} \rightarrow v \text { in } H^{1}\left(\Omega ; \mathbb{R}^{3}\right), \\
\frac{-1}{f_{0}^{\ell} \gamma \cdot n d x_{1}} f_{\omega} \frac{g_{1}^{\varepsilon} \cdot u^{\varepsilon}}{\delta_{\varepsilon}} d a \rightarrow \Theta \text { in } H^{1}(0, L),
\end{gathered}
$$

with $\bar{v}^{\varepsilon}:=\frac{u^{\varepsilon}-u_{3}^{\varepsilon} e_{3}}{\delta_{\varepsilon} / \varepsilon}$ and $v_{3}^{\varepsilon}:=\frac{u_{3}^{\varepsilon}}{\delta_{\varepsilon}}$, and

$$
\limsup _{\varepsilon \rightarrow 0} \frac{J_{\varepsilon}\left(u^{\varepsilon}\right)}{\delta_{\varepsilon}^{2}} \leq J_{\# 0}(\Theta, v) .
$$

Proof. If $(\Theta, v) \notin \mathcal{A}_{\#}$ there is nothing to prove. Let $(\Theta, v) \in \mathcal{A}_{\#}$ and set

$$
E^{\mathrm{opt}}:=\mathbb{E}_{0} \mathbb{E}_{00} E^{K}\left(\partial_{3} \Theta, \partial_{3} v_{3}\right)
$$

be the optimal strain associated to the pair $(\Theta, v)$. Consider the functional

$$
\mathcal{R}_{\varepsilon}(u):=\int_{\Omega} \mathbb{C}(x)\left(\frac{E^{\varepsilon} u}{\delta_{\varepsilon}}-E^{\mathrm{opt}}\right) \cdot\left(\frac{E^{\varepsilon} u}{\delta_{\varepsilon}}-E^{\mathrm{opt}}\right) \sqrt{g^{\varepsilon}} d x,
$$

and let $u^{\varepsilon}$ be the minimizer, i.e.,

$$
\mathcal{R}_{\varepsilon}\left(u^{\varepsilon}\right)=\min _{u \in H_{\# d n}^{1}\left(\Omega ; \mathbb{R}^{3}\right)} \mathcal{R}_{\varepsilon}(u)
$$

Then, $u^{\varepsilon}$ satisfies the following problem

$$
\int_{\Omega} \mathbb{C}(x)\left(\frac{E^{\varepsilon} u^{\varepsilon}}{\delta_{\varepsilon}}-E^{\mathrm{opt}}\right) \cdot \frac{E^{\varepsilon} \psi^{\varepsilon}}{\delta_{\varepsilon}} \sqrt{g^{\varepsilon}} d x=0 \quad \forall \psi^{\varepsilon} \in H_{\# d n}^{1}\left(\Omega ; \mathbb{R}^{3}\right) .
$$

By taking $\psi^{\varepsilon}=u^{\varepsilon}$ into (66) and substituting the resulting equation into the expression of $\mathcal{R}_{\varepsilon}\left(u^{\varepsilon}\right)$ we find

$$
\mathcal{R}_{\varepsilon}\left(u^{\varepsilon}\right)=\int_{\Omega} \mathbb{C}(x) E^{\mathrm{opt}} \cdot E^{\mathrm{opt}} \sqrt{g^{\varepsilon}} d x-\int_{\Omega} \mathbb{C}(x) \frac{E^{\varepsilon} u^{\varepsilon}}{\delta_{\varepsilon}} \cdot \frac{E^{\varepsilon} u^{\varepsilon}}{\delta_{\varepsilon}} \sqrt{g^{\varepsilon}} d x,
$$

from which we deduce that

$$
\int_{\Omega} \mathbb{C}(x) E^{\mathrm{opt}} \cdot E^{\mathrm{opt}} \sqrt{g^{\varepsilon}} d x \geq \int_{\Omega} \mathbb{C}(x) \frac{E^{\varepsilon} u^{\varepsilon}}{\delta_{\varepsilon}} \cdot \frac{E^{\varepsilon} u^{\varepsilon}}{\delta_{\varepsilon}} \sqrt{g^{\varepsilon}} d x=2 \frac{J_{\varepsilon}\left(u^{\varepsilon}\right)}{\delta_{\varepsilon}^{2}} .
$$


Since the term on the left hand side is bounded, then the sequence on the right is also bounded. Therefore, by Theorems 4.2 and 4.5, we conclude that there is a subsequence (not relabelled) of $u^{\varepsilon}$ such that

$$
\begin{gathered}
\bar{v}^{\varepsilon}+v_{3}^{\varepsilon} e_{3}-\tilde{v} \text { in } H^{1}\left(\Omega ; \mathbb{R}^{3}\right), \\
\frac{-1}{f_{0}^{\ell} \gamma \cdot n d x_{1}} f_{\omega} \frac{g_{1}^{\varepsilon} \cdot u^{\varepsilon}}{\delta_{\varepsilon}} d a \rightarrow \tilde{\Theta} \text { in } H^{1}(0, L),
\end{gathered}
$$

where the rescaled displacements $v^{\varepsilon}$ are defined by means of $(21)$ and $(\tilde{\Theta}, \tilde{v}) \in \mathcal{A}_{\#}$.

Also, there exists $\tilde{E} \in L^{2}\left(\Omega ; \mathbb{R}_{\mathrm{sym}}^{3 \times 3}\right)$ such that, up to a subsequence,

$$
\frac{E^{\varepsilon} u^{\varepsilon}}{\delta_{\varepsilon}} \rightarrow \tilde{E} \text { in } L^{2}\left(\Omega ; \mathbb{R}^{3 \times 3}\right)
$$

with $\tilde{E}$ related to $(\tilde{\Theta}, \tilde{v})$ as stated in Lemma 4.6 .

We first prove that $\tilde{E} \in \mathbb{E}_{0} \mathcal{S}^{\perp}$.

Let us choose

$\psi^{\varepsilon}:=\delta_{\varepsilon}^{2}\left(\int_{-h / 2}^{x_{2}} \varphi_{1}\left(x_{1}, \zeta, x_{3}\right) d \zeta t+\int_{-h / 2}^{x_{2}} \varphi_{2}\left(x_{1}, \zeta, x_{3}\right) d \zeta n+\int_{-h / 2}^{x_{2}} \varphi_{3}\left(x_{1}, \zeta, x_{3}\right) d \zeta e_{3}\right)$ with $\varphi_{i} \in C_{0}^{\infty}(\Omega)$, for $i=1,2,3$. Then, $\psi^{\varepsilon} \in H_{\# d n}^{1}\left(\Omega ; \mathbb{R}^{3}\right)$ and

$$
\frac{E^{\varepsilon} \psi^{\varepsilon}}{\delta_{\varepsilon}} \rightarrow \varphi_{1} t \odot n+\varphi_{2} n \odot n+\varphi_{3} e_{3} \odot n
$$

uniformly. Thus, passing to the limit in (66), we get

$$
\int_{\Omega} \mathbb{C}(x)\left(\tilde{E}-E^{\mathrm{opt}}\right) \cdot\left(\varphi_{1} t \odot n+\varphi_{2} n \odot n+\varphi_{3} e_{3} \odot n\right) d x=0 \quad \forall \varphi \in C_{0}^{\infty}\left(\Omega ; \mathbb{R}^{3}\right) .
$$

It follows that

$$
\begin{aligned}
& \mathbb{C}(x)\left(\tilde{E}-E^{\mathrm{opt}}\right) \cdot t \odot n=0, \\
& \mathbb{C}(x)\left(\tilde{E}-E^{\mathrm{opt}}\right) \cdot n \odot n=0, \\
& \mathbb{C}(x)\left(\tilde{E}-E^{\mathrm{opt}}\right) \cdot n \odot e_{3}=0 .
\end{aligned}
$$

Recalling (64) and using (46) twice we deduce that

$$
\tilde{E} \in \mathbb{E}_{0} \mathcal{S}^{\perp}, \quad \text { i.e., } \quad \tilde{E}=\mathbb{E}_{0} \tilde{E}^{\perp}
$$

with $\tilde{E}^{\perp} \in \mathscr{E}^{\perp}$.

We now show that $\tilde{E}=\mathbb{E}_{0} \mathbb{E}_{00} E^{K}\left(\partial_{3} \tilde{\Theta}, \partial_{3} \tilde{v}_{3}\right)$. For any $\varphi_{1}, \varphi_{2} \in C_{0}^{\infty}((0, \ell) \times(0, L))$ such that

$$
\int_{0}^{\ell} \varphi_{2} d x_{1}=0
$$

let

$$
\begin{aligned}
\psi^{\varepsilon}\left(x_{1}, x_{3}\right)=\delta_{\varepsilon} \varepsilon[ & \left(\int_{0}^{x_{1}} \varphi_{1}\left(s, x_{3}\right) d s-x_{1} f_{0}^{\ell} \varphi_{1}\left(s, x_{3}\right) d s\right) t\left(x_{1}\right) \\
& \left.+f_{0}^{\ell} \varphi_{1}\left(s, x_{3}\right) d s \gamma\left(x_{1}\right)+\int_{0}^{x_{1}} \varphi_{2}\left(s, x_{3}\right) d s e_{3}\right],
\end{aligned}
$$

Then, $\psi^{\varepsilon} \in H_{\# d n}^{1}\left(\Omega ; \mathbb{R}^{3}\right)$ and

$$
\frac{E^{\varepsilon} \psi^{\varepsilon}}{\delta_{\varepsilon}} \rightarrow\left(\int_{0}^{x_{1}} \varphi_{1}\left(s, x_{3}\right) d s-x_{1} f_{0}^{\ell} \varphi_{1}\left(s, x_{3}\right) d s\right) \kappa t \odot n+\varphi_{1} t \odot t+\varphi_{2} t \odot e_{3}
$$


uniformly. Thence, if we take account of $(70)_{1}$ and pass to the limit in (66), we deduce that

$$
\int_{\Omega} \mathbb{C}(x)\left(\tilde{E}-E^{\mathrm{opt}}\right) \cdot\left(\varphi_{1} t \odot t+\varphi_{2} t \odot e_{3}\right) d x=0
$$

for all $\varphi_{1}, \varphi_{2} \in C_{0}^{\infty}((0, \ell) \times(0, L))$ with $\varphi_{2}$ satisfying the integral constraint (72). Recalling (64) and using Lemma 5.1 we find that

$$
\int_{\Omega} \mathbb{C}(x) \tilde{E} \cdot\left(\varphi_{1} t \odot t+\varphi_{2} t \odot e_{3}\right) d x=0
$$

holds, by density, for every $\varphi_{1}, \varphi_{2} \in L^{2}((0, \ell) \times(0, L))$ with $\varphi_{2}$ satisfying the integral constraint (72).

Let us now take $\varphi_{3} \in C_{0}^{\infty}((0, \ell) \times(0, L))$ such that

$$
\int_{0}^{\ell} \varphi_{3} d x_{1}=0 \text { and } \int_{0}^{\ell} n\left(x_{1}\right) \int_{0}^{x_{1}} \varphi_{3}(s, \cdot) d s d x_{1}=0
$$

and set

$$
\phi\left(x_{1}, x_{3}\right):=\int_{0}^{x_{1}} \varphi_{3}\left(s, x_{3}\right) d s
$$

and

$$
\psi^{\varepsilon}:=\delta_{\varepsilon} \varepsilon x_{2} \phi t-\varepsilon^{2} \int_{0}^{x_{1}} \phi n d x_{1} .
$$

Then $\psi^{\varepsilon} \in H_{\# d n}^{1}\left(\Omega ; \mathbb{R}^{3}\right)$ and we have

$$
\frac{E^{\varepsilon} \psi^{\varepsilon}}{\delta_{\varepsilon}} \rightarrow x_{2} \partial_{1} \phi t \odot t-\mathfrak{s} n \cdot \int_{0}^{x_{1}} \partial_{3} \phi n d x_{1} n \odot e_{3}
$$

uniformly. By passing to the limit in (66) and using $(70)_{3}$ we deduce that

$$
\int_{\Omega} x_{2} \mathbb{C}(x)\left(\tilde{E}-E^{\mathrm{opt}}\right) \cdot \varphi_{3} t \odot t d x=0 .
$$

Again, recalling (64) and using Lemma 5.1 we find that

$$
\int_{\Omega} x_{2} \mathbb{C}(x) \tilde{E} \cdot \varphi_{3} t \odot t d x=0
$$

holds, by density, for every $\varphi_{3} \in L^{2}((0, \ell) \times(0, L))$ satisfying the constraints (74).

From (71), (73), (75), and Lemma 5.1 we deduce that

$$
\tilde{E}=\mathbb{E}_{0} \mathbb{E}_{00} E^{K}\left(\partial_{3} \tilde{\Theta}, \partial_{3} \tilde{v}_{3}\right) .
$$

From (64), (76) and the linearity of $E^{K}(\cdot, \cdot)$ and of the operators $\mathbb{E}_{0}$ and $\mathbb{E}_{00}$ it follows that

$$
\tilde{E}-E^{\mathrm{opt}}=\mathbb{E}_{0} \mathbb{E}_{00} E^{K}\left(\partial_{3}(\tilde{\Theta}-\Theta), \partial_{3}\left(\tilde{v}_{3}-v_{3}\right)\right) .
$$

Finally, we show that $(\Theta, v)=(\tilde{\Theta}, \tilde{v})$.

Let $\bar{\zeta} \in H_{d n}^{2}\left(0, L ; \mathbb{R}^{2} \times\{0\}\right), \zeta_{3} \in H_{d n}^{1}(0, L)$ and $\phi \in H_{d n}^{2}(0, L)$. Set

$$
\bar{\psi}^{\varepsilon}:=\frac{\delta_{\varepsilon}}{\varepsilon} \bar{\zeta}+\delta_{\varepsilon} \phi e_{3} \wedge \gamma-\frac{\delta_{\varepsilon}^{2}}{\varepsilon} x_{2} \phi t
$$

and

$$
\psi_{3}^{\varepsilon}:=\delta_{\varepsilon} \zeta_{3}-\delta_{\varepsilon} \partial_{3} \bar{\zeta} \cdot\left(\gamma+\frac{\delta_{\varepsilon}}{\varepsilon} x_{2} n\right)-\delta_{\varepsilon}^{2} \partial_{3} \phi x_{2} t \cdot \gamma
$$


Setting $\psi^{\varepsilon}:=\bar{\psi}^{\varepsilon}+\psi_{3}^{\varepsilon} e_{3} \in H_{\# d n}^{1}\left(\Omega ; \mathbb{R}^{3}\right)$ we find that

$$
\frac{E^{\varepsilon} \psi^{\varepsilon}}{\delta_{\varepsilon}} \rightarrow-n \cdot \gamma \partial_{3} \phi t \odot e_{3}+\partial_{3}\left(\zeta_{3}-\partial_{3} \bar{\zeta} \cdot \gamma\right) e_{3} \odot e_{3}
$$

uniformly. Therefore, by passing to the limit in (66) and taking (77) into account we deduce that

$$
\int_{\Omega} \mathbb{C E}_{0} \mathbb{E}_{00} E^{K}\left(\partial_{3}(\tilde{\Theta}-\Theta), \partial_{3}\left(\tilde{v}_{3}-v_{3}\right)\right) \cdot\left(-n \cdot \gamma \partial_{3} \phi t \odot e_{3}+\partial_{3} w_{3} e_{3} \odot e_{3}\right) d x=0
$$

where

$$
w_{3}:=\zeta_{3}-\partial_{3} \bar{\zeta} \cdot \gamma \text { and } w:=\bar{\zeta}+w_{3} e_{3} .
$$

Equation (78) holds true for every $w$ as above and, by density, for every $\phi \in$ $H_{d n}^{1}(0, L)$, that is: it holds for every $(\phi, w) \in \mathcal{A}_{\#}$. Recalling the definition (52) of $E^{K}$, and using (56), we may rewrite (78) as

$$
\int_{\Omega} \mathbb{C E}_{0} \mathbb{E}_{00} E^{K}\left(\partial_{3}(\tilde{\Theta}-\Theta), \partial_{3}\left(\tilde{v}_{3}-v_{3}\right)\right) \cdot \mathbb{E}_{0} \mathbb{E}_{00} E^{K}\left(\partial_{3} \phi, \partial_{3} w_{3}\right) d x=0 .
$$

Taking $\phi=\tilde{\Theta}-\Theta$ and $w_{3}=\tilde{v}_{3}-v_{3}$ we find

$$
\int_{\Omega} \mathbb{C E}_{0} \mathbb{E}_{00} E^{K}\left(\partial_{3}(\tilde{\Theta}-\Theta), \partial_{3}\left(\tilde{v}_{3}-v_{3}\right)\right) \cdot \mathbb{E}_{0} \mathbb{E}_{00} E^{K}\left(\partial_{3}(\tilde{\Theta}-\Theta), \partial_{3}\left(\tilde{v}_{3}-v_{3}\right)\right) d x=0,
$$

from which we deduce that

$$
\mathbb{E}_{0} \mathbb{E}_{00} E^{K}\left(\partial_{3}(\tilde{\Theta}-\Theta), \partial_{3}\left(\tilde{v}_{3}-v_{3}\right)\right)=0
$$

since $\mathbb{C}$ is positive definite. Observing that

$$
\mathbb{E}_{0} \mathbb{E}_{00} E^{K}\left(\partial_{3}(\tilde{\Theta}-\Theta), \partial_{3}\left(\tilde{v}_{3}-v_{3}\right)\right) \cdot e_{3} \otimes e_{3}=\partial_{3}\left(\tilde{v}_{3}-v_{3}\right),
$$

from (79) it follows that $\partial_{3}\left(\tilde{v}_{3}-v_{3}\right)=0$. This, and the boundary condition $\tilde{v}_{3}=$ $v_{3}=0$ at $x_{3}=0$, imply that $\tilde{v}_{3}=v_{3}$. By the definition (62) of $\mathcal{A}_{\#}$ we have also

$$
\tilde{v}=v .
$$

Indeed, by (62) the following relations hold true

$$
\left\{\begin{array}{l}
v=\bar{m}+v_{3} e_{3}, \\
\tilde{v}=\tilde{\bar{m}}+\tilde{v}_{3} e_{3}, \\
v_{3}=m_{3}-\partial_{3} \bar{m} \cdot\left(\gamma-\gamma_{G}\right), \\
\tilde{v}_{3}=\tilde{m}_{3}-\partial_{3} \tilde{\bar{m}} \cdot\left(\gamma-\gamma_{G}\right) .
\end{array}\right.
$$

By integrating the last two in $x_{1} \in[0, \ell]$ and using the fact that $\tilde{v}_{3}=v_{3}$ we obtain $m_{3}=\tilde{m}_{3}$, and hence

$$
\partial_{3} \bar{m} \cdot\left(\gamma-\gamma_{G}\right)=\partial_{3} \tilde{\bar{m}} \cdot\left(\gamma-\gamma_{G}\right) .
$$

Since $\gamma$ is not a segment, and using the boundary conditions, it follows that $\bar{m}=\tilde{\bar{m}}$, and hence (80).

From (53) and (79) we find

$$
0=\mathbb{E}_{0} \mathbb{E}_{00} E^{K}\left(\partial_{3}(\tilde{\Theta}-\Theta), \partial_{3}\left(\tilde{v}_{3}-v_{3}\right)\right) \cdot t \odot e_{3}=\xi-\frac{1}{2} \gamma \cdot n \partial_{3}(\tilde{\Theta}-\Theta),
$$

where $\xi \in L^{2}((0, \ell) \times(0, L))$ is such that $\int_{0}^{\ell} \xi d x_{1}=0$. Thus, integrating the above equation over $(0, \ell)$ we deduce that $\partial_{3}(\tilde{\Theta}-\Theta)=0$ and, because of the boundary condition, that

$$
\tilde{\Theta}=\Theta
$$


Since $(\tilde{\Theta}, \tilde{v})=(\Theta, v)$ we have that the sequence of minimizers $u^{\varepsilon}$ generates two sequences, see (68) and (69), as in the statement of the theorem. Moreover from (67) we find

$$
\limsup _{\varepsilon \rightarrow 0} \frac{J_{\varepsilon}\left(u^{\varepsilon}\right)}{\delta_{\varepsilon}^{2}} \leq \limsup _{\varepsilon \rightarrow 0} \frac{1}{2} \int_{\Omega} \mathbb{C}(x) E^{\mathrm{opt}} \cdot E^{\mathrm{opt}} \sqrt{g^{\varepsilon}} d x=\int_{\Omega} f_{00}\left(x, \partial_{3} \Theta, \partial_{3} v_{3}\right) d x,
$$

where we used (64) and (57).

\section{The Reduced Energy Density associated to $f_{00}$}

The aim of this section is to show that the $\Gamma$-limit functional admits a local representation, that is, we shall give a pointwise characterization of the map $f_{00}$ introduced in (57). This will be achieved by localizing the stationarity conditions associated with the minimization problem (54).

Let $a \in L^{2}(0, L)$ and $b \in L^{2}((0, \ell) \times(0, L))$. From Lemma 5.1 with $\varphi_{2}=\varphi_{3}=0$ we find

$$
\left\langle\mathbb{C E}_{0} \mathbb{E}_{00} E^{K}(a, b)\right\rangle \cdot t \otimes t=0,
$$

where, as in [1], we have denoted by

$$
\langle\cdot\rangle:=f_{-h / 2}^{h / 2} \cdot d x_{2}
$$

the integral average over the $x_{2}$ variable. By means of (47), (48), and (53) we may rewrite $(82)$ as

$$
\left\langle\mathbb{C}_{11}\right\rangle \eta_{1}^{\mathrm{opt}}+\left\langle\mathbb{C}_{12}\right\rangle \eta_{2}^{\mathrm{opt}}+\left\langle x_{2} \mathbb{\complement}_{11}\right\rangle \eta_{3}^{\mathrm{opt}}=\frac{1}{2}\left\langle\mathbb{c}_{12}\right\rangle \gamma \cdot n a-\left\langle\mathbb{C}_{13}\right\rangle b .
$$

Still from Lemma 5.1, by taking $\varphi_{1}=\varphi_{3}=0$ we deduce that there exists a scalar function $q \in L^{2}(0, L)$ such that

$$
\left\langle\mathbb{C E}_{0} \mathbb{E}_{00} E^{K}(a, b)\right\rangle \cdot t \otimes e_{3}=q,
$$

or, in expanded form,

$$
\left\langle\mathbb{c}_{12}\right\rangle \eta_{1}^{\mathrm{opt}}+\left\langle\mathbb{C}_{22}\right\rangle \eta_{2}^{\mathrm{opt}}+\left\langle x_{2} \mathbb{\mathbb { C }}_{12}\right\rangle \eta_{3}^{\mathrm{opt}}=\frac{1}{2}\left\langle\mathbb{c}_{22}\right\rangle \gamma \cdot n a-\left\langle\mathbb{C}_{23}\right\rangle b+q .
$$

This equation is the consequence of a standard result recalled in the next lemma.

Lemma 7.1. Let $F \in L^{2}((0, \ell) \times(0, L))$ be such that

$$
\int_{(0, \ell) \times(0, L)} F \psi d x=0
$$

for every $\psi \in L^{2}((0, \ell) \times(0, L))$ with $\int_{0}^{\ell} \psi\left(x_{1}, x_{3}\right) d x_{1}=0$ for a.e. $x_{3} \in(0, L)$. Then, $\partial_{1} F=0$ in the sense of distributions.

Proof. Let $\varphi \in C_{0}^{\infty}((0, \ell) \times(0, L))$ and $\psi:=\partial_{1} \varphi$. Then, $\psi$ satisfies the constraint stated in the lemma and, in the sense of distributions,

$$
\partial_{1} F(\varphi)=-\int_{(0, \ell) \times(0, L)} F \psi d x=0 .
$$

The claim follows by the arbitrariness of $\varphi$. 
To characterize the stationarity condition with respect to $\eta_{3}$, we use a Lagrange multipliers argument in order to handle the double constraint, see for instance [14], Section 4.14.

Let $\mathcal{F}: L^{2}\left((0, L) ; H_{0}^{1}(0, \ell)\right) \rightarrow \mathbb{R}$ and $\mathcal{G}: L^{2}\left((0, L) ; H_{0}^{1}(0, \ell)\right) \rightarrow L^{2}\left((0, L) ; \mathbb{R}^{2} \times\right.$ $\{0\})$ be defined by

$$
\mathcal{F}(\Phi):=\int_{\Omega} f_{0}\left(x, x_{2} \partial_{1} \Phi+\eta_{1}^{\mathrm{opt}}, \eta_{2}^{\mathrm{opt}}-\frac{1}{2} \gamma \cdot n a, b\right) d x
$$

and

$$
\mathcal{G}(\Phi):=\int_{0}^{\ell} \Phi t d x_{1} .
$$

We note that with $\eta_{3}=\partial_{1} \Phi$ the constraints $(51)_{2,3}$ hold if and only if $\mathcal{G}(\Phi)=0$.

Let

$$
\Phi^{\mathrm{opt}}\left(x_{1}, x_{3}\right):=\int_{0}^{x_{1}} \eta_{3}^{\mathrm{opt}} d x_{1} .
$$

Since $\mathcal{G}$ is continuous, differentiable and its Frechét derivative $\mathcal{G}^{\prime}=\mathcal{G}$ is a surjective map from $L^{2}\left((0, L) ; H_{0}^{1}(0, \ell)\right)$ onto $L^{2}\left((0, L) ; \mathbb{R}^{2} \times\{0\}\right)$, as is shown in the Appendix, it follows that there is a Lagrange multiplier $y^{*}$ in the dual of $L^{2}\left((0, L) ; \mathbb{R}^{2} \times\{0\}\right)$ such that at the stationarity point we have

$$
\mathcal{F}^{\prime}\left(\Phi^{\mathrm{opt}}\right)[\delta \Phi]+y^{*} \mathcal{G}^{\prime}[\delta \Phi]=0 \quad \forall \delta \Phi \in L^{2}\left((0, L) ; H_{0}^{1}(0, \ell)\right) .
$$

By identifying the space $L^{2}\left((0, L) ; \mathbb{R}^{2} \times\{0\}\right)$ with its dual, this equation can be written as

$$
\int_{0}^{L} \int_{0}^{\ell}\left\langle\frac{\partial f_{0}}{\partial E_{11}} x_{2}\right\rangle \partial_{1} \delta \Phi+y^{*} \cdot t \delta \Phi d x_{1} d x_{3}=0 \quad \forall \delta \Phi \in L^{2}\left((0, L) ; H_{0}^{1}(0, \ell)\right),
$$

where $\frac{\partial f_{0}}{\partial E_{11}}$ is evaluated in $\left(x, x_{2} \partial_{1} \Phi^{\mathrm{opt}}+\eta_{1}^{\mathrm{opt}}, \eta_{2}^{\mathrm{opt}}-\frac{1}{2} \gamma \cdot n a, b\right)$. It follows that the stationarity condition is

$$
\partial_{1}\left\langle\frac{\partial f_{0}}{\partial E_{11}} x_{2}\right\rangle-y^{*} \cdot t=0
$$

Recalling that $t=\partial_{1} \gamma$ and $y^{*}$ does not depend upon $x_{1}$, by integrating we arrive at

$$
\left\langle\frac{\partial f_{0}}{\partial E_{11}} x_{2}\right\rangle=y^{*} \cdot\left(\gamma-\gamma_{G}\right)+c
$$

with $y^{*} \in L^{2}\left((0, L) ; \mathbb{R}^{2} \times\{0\}\right)$ and $c \in L^{2}(0, L)$.

We may rewrite $(83),(84)$, and (85) in expanded form as (86)

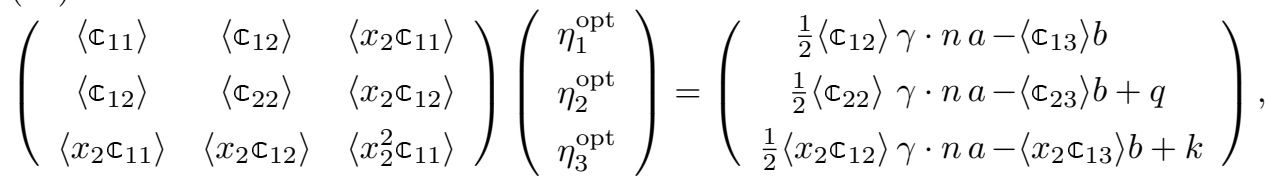

with

$$
k:=y^{*} \cdot\left(\gamma-\gamma_{G}\right)+c .
$$

The matrix of coefficients in (86) is invertible, in fact uniformly positive definite, by the argument following equation (61) in [1]. Thus, system (86) provides $\left(\eta_{1}^{\mathrm{opt}}, \eta_{2}^{\mathrm{opt}}, \eta_{3}^{\mathrm{opt}}\right)$ in terms of $(a, b, q, k)$. By imposing the constraints $(51)$ we then find $q$ and $k$ in terms of the pair $(a, b)$. To see this, let

$$
r_{0}:=\left(\left\langle\mathbb{C}_{13}\right\rangle,\left\langle\mathbb{C}_{23}\right\rangle,\left\langle x_{2} \mathbb{C}_{13}\right\rangle\right)^{T}, \quad s_{0}:=\left(\left\langle\mathbb{C}_{12}\right\rangle,\left\langle\mathbb{C}_{22}\right\rangle,\left\langle x_{2} \mathbb{C}_{12}\right\rangle\right)^{T}
$$


and

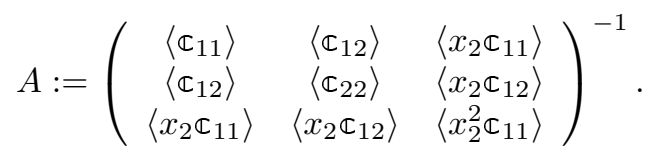

Then, $(51)_{1}$ and $(51)_{2}$ respectively require that

$$
\int_{0}^{\ell} A_{22} q+A_{23}\left(c+\left(\gamma-\gamma_{G}\right) \cdot y^{*}\right) d x_{1}=\int_{0}^{\ell}\left(A r_{0}\right)_{2} b-\frac{1}{2}\left(A s_{0}\right)_{2} \gamma \cdot n a d x_{1},
$$

$$
\int_{0}^{\ell} A_{32} q+A_{33}\left(c+\left(\gamma-\gamma_{G}\right) \cdot y^{*}\right) d x_{1}=\int_{0}^{\ell}\left(A r_{0}\right)_{3} b-\frac{1}{2}\left(A s_{0}\right)_{3} \gamma \cdot n a d x_{1} .
$$

As a matter of fact, $\left(A s_{0}\right)_{2}=1$ and $\left(A s_{0}\right)_{3}=0$, because $s_{0}$ is the second column of the matrix $A^{-1}$. Thus, equations (88) take the form

$$
\int_{0}^{\ell} A_{22} q+A_{23}\left(c+\left(\gamma-\gamma_{G}\right) \cdot y^{*}\right) d x_{1}=\int_{0}^{\ell}\left(A r_{0}\right)_{2} b-\frac{1}{2} \gamma \cdot n a d x_{1},
$$

$$
\int_{0}^{\ell} A_{32} q+A_{33}\left(c+\left(\gamma-\gamma_{G}\right) \cdot y^{*}\right) d x_{1}=\int_{0}^{\ell}\left(A r_{0}\right)_{3} b d x_{1} .
$$

Moreover, from (86) we find

$$
\eta_{3}^{\mathrm{opt}}=-\left(A r_{0}\right)_{3} b+A_{32} q+A_{33}\left(c+\left(\gamma-\gamma_{G}\right) \cdot y^{*}\right) .
$$

Observing that $t=\partial_{1}\left(\gamma-\gamma_{G}\right)$, the constraint $(51)_{3}$ can be written as

$$
\int_{0}^{\ell} \partial_{1}\left(\gamma-\gamma_{G}\right) \int_{0}^{x_{1}} \eta_{3}^{\mathrm{opt}} d s d x_{1}=0
$$

Thus, after an integration by parts and taking account of $(51)_{2}$ we get

$$
\int_{0}^{\ell} \eta_{3}^{\mathrm{opt}}\left(\gamma-\gamma_{G}\right) d x_{1}=0
$$

Thence, by implementing (90) we obtain

$$
\int_{0}^{\ell} A_{32}\left(\gamma-\gamma_{G}\right) q+A_{33}\left(\gamma-\gamma_{G}\right)\left(c+\left(\gamma-\gamma_{G}\right) \cdot y^{*}\right) d x_{1}=\int_{0}^{\ell}\left(A r_{0}\right)_{3}\left(\gamma-\gamma_{G}\right) b d x_{1} .
$$

Equations (89) and (91) can be rewritten as

$$
\mathcal{A}\left(q, c, y_{1}^{*}, y_{2}^{*}\right)^{T}=F(a, b),
$$

where

$$
\begin{aligned}
& \mathcal{A}:= \\
& \left(\begin{array}{llll}
\int_{0}^{\ell} A_{22} d x_{1} & \int_{0}^{\ell} A_{23} d x_{1} & \int_{0}^{\ell} A_{23}\left(\gamma-\gamma_{G}\right)_{1} d x_{1} & \int_{0}^{\ell} A_{23}\left(\gamma-\gamma_{G}\right)_{2} d x_{1} \\
& \int_{0}^{\ell} A_{33} d x_{1} & \int_{0}^{\ell} A_{33}\left(\gamma-\gamma_{G}\right)_{1} d x_{1} & \int_{0}^{\ell} A_{33}\left(\gamma-\gamma_{G}\right)_{2} d x_{1} \\
& & \int_{0}^{\ell} A_{33}\left(\gamma-\gamma_{G}\right)_{1}^{2} d x_{1} & \int_{0}^{\ell} A_{33}\left(\gamma-\gamma_{G}\right)_{1}\left(\gamma-\gamma_{G}\right)_{2} d x_{1} \\
& & & \int_{0}^{\ell} A_{33}\left(\gamma-\gamma_{G}\right)_{2}^{2} d x_{1}
\end{array}\right)
\end{aligned}
$$


and

$$
\begin{aligned}
F(a, b):= & \left(\int_{0}^{\ell}\left(A r_{0}\right)_{2} b-\frac{1}{2} \gamma \cdot n a d x_{1}, \int_{0}^{\ell}\left(A r_{0}\right)_{3} b d x_{1},\right. \\
& \left.\int_{0}^{\ell}\left(A r_{0}\right)_{3}\left(\gamma-\gamma_{G}\right)_{1} b d x_{1}, \int_{0}^{\ell}\left(A r_{0}\right)_{3}\left(\gamma-\gamma_{G}\right)_{2} b d x_{1}\right)^{T} .
\end{aligned}
$$

The matrix $\mathcal{A}$ is positive definite. Indeed, for $z \in \mathbb{R}^{4}$ we have

$$
\mathcal{A} z \cdot z=\int_{0}^{\ell}\left(\begin{array}{cc}
A_{22} & A_{23} \\
A_{32} & A_{33}
\end{array}\right)\left(\begin{array}{c}
z_{1} \\
z_{2}+\left(\gamma-\gamma_{G}\right) \cdot z^{*}
\end{array}\right) \cdot\left(\begin{array}{c}
z_{1} \\
z_{2}+\left(\gamma-\gamma_{G}\right) \cdot z^{*}
\end{array}\right) d x_{1},
$$

where $z^{*}=\left(z_{3}, z_{4}\right)$. Since $A$ is uniformly positive definite, there is a constant $\sigma>0$ such that

$$
\begin{aligned}
\mathcal{A} z \cdot z & \geq \sigma \int_{0}^{\ell}\left|\left(\begin{array}{c}
z_{1} \\
z_{2}+\left(\gamma-\gamma_{G}\right) \cdot z^{*}
\end{array}\right)\right|^{2} d x_{1} \\
& =\sigma \int_{0}^{\ell} z_{1}^{2}+z_{2}^{2}+\left|\left(\gamma-\gamma_{G}\right) \cdot z^{*}\right|^{2} d x_{1}
\end{aligned}
$$

since $\int_{0}^{\ell}\left(\gamma-\gamma_{G}\right) \cdot z^{*} d x_{1}=0$. Thus,

$$
\begin{aligned}
\mathcal{A} z \cdot z & \geq \sigma\left(\ell\left(z_{1}^{2}+z_{2}^{2}\right)+\int_{0}^{\ell}\left(\gamma-\gamma_{G}\right) \otimes\left(\gamma-\gamma_{G}\right) d x_{1} z^{*} \cdot z^{*}\right) \\
& \geq \sigma\left(\ell\left(z_{1}^{2}+z_{2}^{2}\right)+I_{\min }\left|z^{*}\right|^{2}\right)
\end{aligned}
$$

where $I_{\min }$ denotes the minimum eigenvalue of the tensor of inertia of the midline curve $\gamma$ that appears in the first line.

From system (92) we deduce

$$
\left(q, c, y_{1}^{*}, y_{2}^{*}\right)^{T}=\mathcal{A}^{-1} F(a, b),
$$

and hence, from system (86) we find

$$
\begin{aligned}
& \eta_{1}^{\mathrm{opt}}=-\left(A r_{0}\right)_{1} b+A_{12}\left(\mathcal{A}^{-1} F(a, b)\right)_{1}+A_{13} k, \\
& \eta_{2}^{\mathrm{opt}}=\frac{1}{2} \gamma \cdot n a-\left(A r_{0}\right)_{2} b+A_{22}\left(\mathcal{A}^{-1} F(a, b)\right)_{1}+A_{23} k, \\
& \eta_{3}^{\mathrm{opt}}=-\left(A r_{0}\right)_{3} b+A_{32}\left(\mathcal{A}^{-1} F(a, b)\right)_{1}+A_{33} k,
\end{aligned}
$$

where

$$
k=\left(\mathcal{A}^{-1} F(a, b)\right)_{2}+\left(\gamma-\gamma_{G}\right) \cdot\left(\left(\mathcal{A}^{-1} F(a, b)\right)_{3},\left(\mathcal{A}^{-1} F(a, b)\right)_{4}, 0\right) .
$$

These equations deliver the optimal $\eta$ 's in terms of $a \in L^{2}(0, L)$ and $b \in L^{2}((0, \ell) \times$ $(0, L))$. It is possible to make these relations pointwise (local) by restricting $b$ to the subclass of functions of $L^{2}((0, \ell) \times(0, L))$ having the same structure of $\partial_{3} v_{3}$, that is those of the form

$$
b=b_{0}-\left(\gamma-\gamma_{G}\right) \cdot b^{*}, \quad b^{*}:=\left(b_{1}, b_{2}, 0\right)
$$

with $b_{0}, b_{1}, b_{2} \in L^{2}(0, L)$. Indeed, we can define a function

$$
g: \Omega \times \mathbb{R}^{4} \rightarrow \mathbb{R}^{3}
$$

such that, for $b$ as in (96), we can write (95) as

$$
\eta_{i}^{\mathrm{opt}}=g_{i}\left(x, a, b_{0}, b_{1}, b_{2}\right)
$$


By means of this function $g$ we define the energy density $\bar{f}_{00}: \Omega \times \mathbb{R}^{4} \rightarrow \mathbb{R}$ as

$$
\bar{f}_{00}(x, z):=f_{0}\left(x, x_{2} g_{3}(x, z)+g_{1}(x, z), g_{2}(x, z)-\frac{1}{2} \gamma \cdot n z_{1}, z_{2}-\left(\gamma-\gamma_{G}\right) \cdot z^{*}\right),
$$

with $z^{*}:=\left(z_{3}, z_{4}, 0\right)$. Taking into account (58), we may rewrite the energy appearing in the $\Gamma$-limit as

$$
f_{00}\left(x, \partial_{3} \Theta, \partial_{3} v_{3}\right)=\bar{f}_{00}\left(x, \partial_{3} \Theta, \partial_{3} m_{3}, \partial_{3}^{2} \bar{m}_{1}, \partial_{3}^{2} \bar{m}_{2}\right),
$$

with

see (62).

$$
v_{3}=m_{3}-\left(\gamma-\gamma_{G}\right) \cdot \partial_{3} \bar{m}
$$

\section{The Case of homogeneous isotropic material}

We conclude with the computation of the strain energy functional of the asymptotic model for a thin-walled beam made of an isotropic and homogeneous elastic material, that is

$$
\mathbb{C} \tilde{E}:=2 \mu \tilde{E}+\lambda(\tilde{E} \cdot I) I
$$

for every symmetric matrix $\tilde{E}$, where $\lambda$ and $\mu$ are the Lamé constants. In this case, from (44), the reduced elastic constants introduced in (48) can be computed as

$$
\mathbb{c}_{11}=\mathbb{C}_{33}=4 \mu \frac{\lambda+\mu}{\lambda+2 \mu}, \quad \mathbb{c}_{22}=4 \mu, \quad \mathbb{c}_{13}=\mathbb{c}_{31}=2 \frac{\lambda \mu}{\lambda+2 \mu},
$$

all others being equal to zero. It follows that the matrix of coefficients in system (86) is diagonal, its inverse $A$ being given by

$$
A=\left[\begin{array}{ccc}
1 / \mathbb{C}_{11} & 0 & 0 \\
0 & 1 / \mathbb{C}_{22} & 0 \\
0 & 0 & 1 /\left\langle x_{2}^{2} \mathbb{C}_{11}\right\rangle
\end{array}\right]
$$

By choosing principal axes of inertia, also the matrix $\mathcal{A}$ defined in (93) is diagonal and

$$
\mathcal{A}^{-1} F(a, b)=\left(-\frac{1}{2} \mathbb{C}_{22} f_{0}^{\ell} \gamma \cdot n d x_{1} a, 0,0,0\right)^{T} .
$$

From (95), by using this expression we may compute the optimal $\eta$ 's, and then, from (97), find

$$
\begin{aligned}
\eta_{1}^{\mathrm{opt}} & =g_{1}=-\nu\left(b_{0}-\left(\gamma-\gamma_{G}\right)_{1} b_{1}-\left(\gamma-\gamma_{G}\right)_{2} b_{2}\right), \\
\eta_{2}^{\mathrm{opt}} & =g_{2}=\frac{1}{2}\left(\gamma \cdot n-f_{0}^{\ell} \gamma \cdot n d x_{1}\right) a, \\
\eta_{3}^{\mathrm{opt}} & =g_{3}=0,
\end{aligned}
$$

where

$$
\nu:=\frac{\lambda}{2(\lambda+\mu)}
$$

is the Poisson ratio. The reduced energy density turns out to be

$$
\bar{f}_{00}(\cdot, z)=\frac{1}{2} \mu\left(f_{0}^{\ell} \gamma \cdot n d x_{1}\right)^{2} z_{1}^{2}+\frac{1}{2} E_{Y}\left(z_{2}-\left(\gamma-\gamma_{G}\right) \cdot\left(z_{3}, z_{4}, 0\right)\right)^{2}
$$

where

is the Young modulus.

$$
E_{Y}:=\frac{\mu(2 \mu+3 \lambda)}{\mu+\lambda}
$$


Let $(\Theta, v) \in \mathcal{A}_{\#}$. Then $\partial_{3} v_{3}$ has the representation

$$
\partial_{3} v_{3}=\partial_{3} m_{3}-\left(\gamma-\gamma_{G}\right) \cdot \partial_{3}^{2} \bar{m}
$$

and from (98) and (63) we deduce that

$$
\begin{aligned}
J_{\# 0}(\Theta, v)= & \frac{1}{2} \mu\left(f_{0}^{\ell} \gamma \cdot n d x_{1}\right)^{2} \int_{\Omega}\left(\partial_{3} \Theta\right)^{2} d x+\frac{1}{2} E_{Y} \int_{\Omega}\left(\partial_{3} v_{3}\right)^{2} d x \\
= & \frac{1}{2} \mu h \ell\left(f_{0}^{\ell} \gamma \cdot n d x_{1}\right)^{2} \int_{0}^{L}\left(\partial_{3} \Theta\right)^{2} d x_{3}+\frac{1}{2} E_{Y} h \ell \int_{0}^{L}\left(\partial_{3} m_{3}\right)^{2} d x_{3} \\
& +\frac{1}{2} E_{Y} h J_{2} \int_{0}^{L}\left(\partial_{3}^{2} \bar{m}_{1}\right)^{2} d x_{3}+\frac{1}{2} E_{Y} h J_{1} \int_{0}^{L}\left(\partial_{3}^{2} \bar{m}_{2}\right)^{2} d x_{3},
\end{aligned}
$$

where we set

$$
J_{1}:=\int_{0}^{\ell}\left(\gamma-\gamma_{G}\right)_{2}^{2} d x_{1}, \quad J_{2}:=\int_{0}^{\ell}\left(\gamma-\gamma_{G}\right)_{1}^{2} d x_{1}
$$

Remark 8.1. For the field $(\Theta, v) \in \mathcal{A}_{\#}$ as above, let the optimal strain be denoted by $E^{\mathrm{opt}}:=\mathbb{E}_{0} \mathbb{E}_{00} E^{K}\left(\partial_{3} \Theta, \partial_{3} v_{3}\right)$, see the proof of Theorem 6.2. Then, $\eta_{i}^{\text {opt }}=g_{i}\left(x, \partial_{3} \Theta, \partial_{3} m_{3}, \partial_{3}^{2} \bar{m}_{1}, \partial_{3}^{2} \bar{m}_{2}\right)$, from (100) and (53),

$$
\begin{aligned}
& E_{11}^{\mathrm{opt}}=-\nu \partial_{3} v_{3}, \\
& E_{13}^{\mathrm{opt}}=-\frac{1}{2} f_{0}^{\ell} \gamma \cdot n d x_{1} \partial_{3} \Theta, \\
& E_{33}^{\mathrm{opt}}=\partial_{3} v_{3} .
\end{aligned}
$$

We observe that component $E_{11}^{\text {opt }}$ of the strain is proportional to $E_{33}^{\text {opt }}$ (Poisson effect). From (43) and $(101)_{2}$ we find

$$
\partial_{1} \varphi-\gamma \cdot n \partial_{3} \Theta=2 E_{13}^{\mathrm{opt}}=-f_{0}^{\ell} \gamma \cdot n d x_{1} \partial_{3} \Theta
$$

and, by integration, one obtains

$$
\varphi=\left(\int_{0}^{x_{1}} \gamma \cdot n d x_{1}-\frac{x_{1}}{\ell} \int_{0}^{\ell} \gamma \cdot n d x_{1}\right) \partial_{3} \Theta .
$$

The expression within the square brackets is the sectorial area formula for the warping function in Vlasov's theory, see [8].

In Section 7 we used the theory of Lagrange multipliers with the proviso that the map $\mathcal{G}^{\prime}: L^{2}\left((0, L) ; H_{0}^{1}(0, \ell)\right) \rightarrow L^{2}\left((0, L) ; \mathbb{R}^{2} \times\{0\}\right)$ defined by

$$
\mathcal{G}^{\prime}(\Phi):=\int_{0}^{\ell} \Phi t d x_{1}
$$

was surjective. Here we prove this statement.

It is possible to choose $\varphi^{(1)}, \varphi^{(2)} \in H_{0}^{1}(0, \ell)$ such that the vectors

$$
v^{(1)}=\int_{0}^{\ell} \varphi^{(1)} t d x_{1} \quad \text { and } \quad v^{(2)}=\int_{0}^{\ell} \varphi^{(2)} t d x_{1}
$$

are linearly independent in $\mathbb{R}^{2} \times\{0\}$.

Let $y \in L^{2}\left((0, L) ; \mathbb{R}^{2} \times\{0\}\right)$. We look for a pair of functions $a_{\alpha}=a_{\alpha}\left(x_{3}\right), \alpha=$ 1,2 , such that for

$$
\Phi=a_{1} \varphi^{(1)}+a_{2} \varphi^{(2)}
$$


we have that

$$
\int_{0}^{\ell} \Phi t d x_{1}=y
$$

This is equivalent to require that $a_{1}$ and $a_{2}$ satisfy

$$
a_{1} v^{(1)}+a_{2} v^{(2)}=y .
$$

More explicitly, the above equation takes the form

$$
\left(\begin{array}{ll}
v_{1}^{(1)} & v_{1}^{(2)} \\
v_{2}^{(1)} & v_{2}^{(2)}
\end{array}\right)\left(\begin{array}{l}
a_{1} \\
a_{2}
\end{array}\right)=\left(\begin{array}{l}
y_{1} \\
y_{2}
\end{array}\right) .
$$

Since the matrix of coefficients is invertible, we may write $a_{1}$ and $a_{2}$ as a linear combination of $y_{1}$ and $y_{2}$. Thus $a_{\alpha} \in L^{2}(0, L)$ and hence $\Phi \in L^{2}\left((0, L) ; H_{0}^{1}(0, \ell)\right)$.

Acknowledgements. The work of CD has been supported by a grant of the University of Udine on "Asymptotic methods and models in the mechanics of materials and structures". The work of LF has been partially supported by the INDAMGNAMPA project 2014 "Metodi asintotici per problemi di fratture, delaminazioni e giunzioni". RP is grateful to Regione Autonoma della Sardegna for the support given under the project "Modellazione Multiscala della Meccanica dei Materiali Compositi (M4C)".

\section{REFERENCES}

[1] C Davini, L. Freddi, and R. Paroni, Linear models for composite thin-walled beams by C-convergence. Part I: Open cross-sections, (2014), accepted by SIAM J. Math. Anal.

[2] E. DAvoli, Thin-walld beams with a cross-section of arbitrary geometry: derivation of linear theories starting from 3D nonlinear elasticity, Adv. Calc. Var., 6 (2013), pp. 33-91.

[3] L. Freddi, M. G. Mora, And R. Paroni, Nonlinear thin-walled beams with a rectangular cross-section-Part I, Math. Models Methods Appl. Sci., 22 (2012), pp. 1150016, 34.

[4] L. Freddi, M. G. Mora, And R. Paroni, Nonlinear thin-walled beams with a rectangular cross-section-Part II, Math. Models Methods Appl. Sci., 23 (2013), pp. 743-775.

[5] L. Freddi, A. Morassi, And R. PAROni, Thin-walled beams: the case of the rectangular cross-section, J. Elasticity, 76 (2004), pp. 45-66 (2005).

[6] L. Freddi, A. Morassi, And R. Paroni, Thin-walled beams: a derivation of Vlassov theory via $\Gamma$-convergence, J. Elasticity, 86 (2007), pp. 263-296.

[7] L. Freddi, F. Murat, and R. PARONi, Anisotropic inhomogeneous rectangular thin-walled beams, SIAM J. Math. Anal., 40 (2008/09), pp. 1923-1951.

[8] C. F. Kollbrunner, K. Basler, (1969), Torsion in structures, Berlin-Heidelberg: SpringerVerlag.

[9] L. Librescu, (2006), Thin-walled composite beams. Theory and applications, in Solid Mechanics and Its Applications, G.M.L. Gladwell eds., Dordrecht: Springer-Verlag.

[10] L. PrandtL, Zur Torsion von prismatischen Staben, Phys. Z. 4 (1903), p. 758-770.

[11] S. Timoshenko and J. M. Geer, Theory of Elastic Stability, McGraw-Hill Book, New York, (1961).

[12] V. Z. Vlasov (1961). Thin-Walled Elastic Beams. Israel Program for Scientific Translations. Jerusalem.

[13] V. Volovoi, D. Hodges, C. Cesnik, And B. Popescu, Assessment of beam modeling methods for rotor blade applications, Mathematical and Computer Modelling, 33 (2001), pp. 1099 1112.

[14] E. ZEIDLER (1995). Applied functional analysis: main principles and their applications. Applied mathematical Sciences 109. New York, NY: Springer-Verlag. 